\title{
EVALUATING CONTEXT EFFECTS ON FORENSIC EXAMINERS: POTENTIAL INFLUENCES ON THE PERCEPTION OF EVIDENCE AND EXAMINERS' DECISION- MAKING
}

\author{
by \\ Melanie Taylor \\ Bachelor of Arts (Hons) in Psychology, Memorial University of Newfoundland, 2016
}

\author{
A thesis presented to Ryerson University \\ in partial fulfillment of the \\ requirements for the degree of \\ Master of Arts \\ in the program of \\ Psychology
}

Toronto, Ontario, Canada, 2018

(C) Melanie Taylor, 2018 


\section{AUTHOR'S DECLARATION FOR ELECTRONIC SUBMISSION OF A THESIS}

I hereby declare that I am the sole author of this thesis. This is a true copy of the thesis, including any required final revisions, as accepted by my examiners.

I authorize Ryerson University to lend this thesis to other institutions or individuals for the purpose of scholarly research.

I further authorize Ryerson University to reproduce this thesis by photocopying or by other means. In total or in part, at the request of other institutions or individuals for the purpose of scholarly research.

I understand that my thesis may be made electronically available to the public. 
Evaluating Context Effects on Forensic Examiners: Potential Influences on the Perception of Evidence and Examiners' Decision-Making

Master of Arts (2018)

Melanie Taylor

Psychology

Ryerson University

The current study aimed to investigate whether contextual information affects examiners' perception of fingerprint evidence and/or their decision-making process regarding whether the fingerprints are a match, nonmatch, or if it is inconclusive. Undergraduates $(\mathrm{N}=129)$ were trained in fingerprint examination and subsequently examined two pairs of fingerprints. The time at which contextual information was distributed was manipulated to change what part of the examination could be influenced by the contextual information. It was hypothesized that the contextual information would affect both the examiner's perception of the evidence as well as their decision-making process. Results were partially in line with this hypothesis; though the contextual information presented demonstrated no effect on participants' conclusions, the timing at which the contextual information was presented did. Implications of these findings are discussed. 


\section{Acknowledgements}

First and foremost, I would like to express my sincere appreciation to my supervisor, Dr. Tara Burke, for the role she has played throughout my time at Ryerson University. Her guidance, knowledge, and her open-minded approach of allowing students to find their own interests and specific research areas has allowed for an invaluable experience during the completion of this degree. I also want to thank Dr. Eric Hehman on my supervisory committee. He has provided a different perspective to this research as well as contributed his breadth of knowledge concerning quantitative research methods and statistics. His input is always graciously accepted. 


\section{Table of Contents}

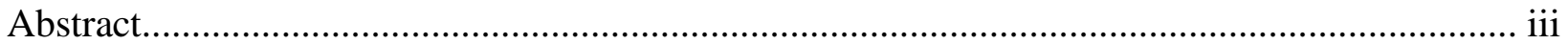

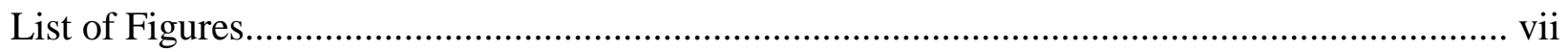

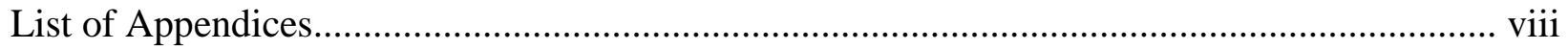

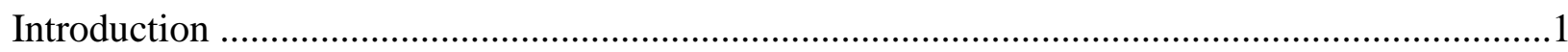

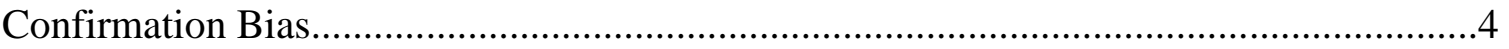

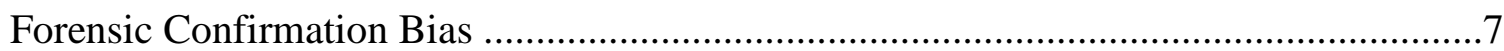

Forensic Confirmation Bias and Forensic Science..................................................

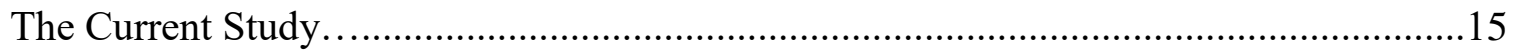

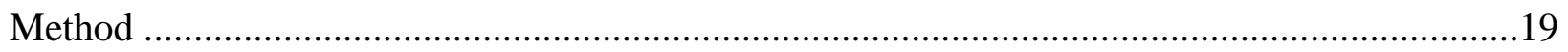

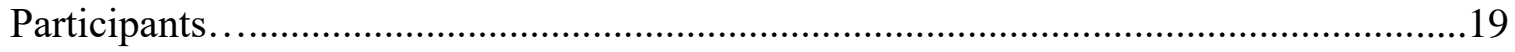

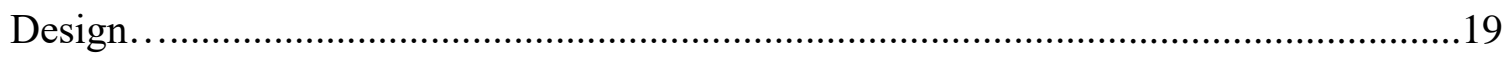

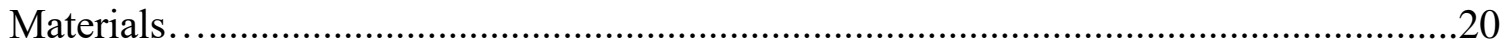

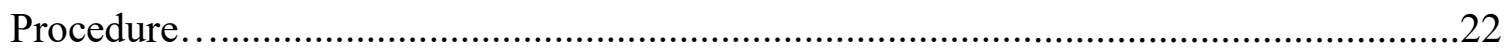

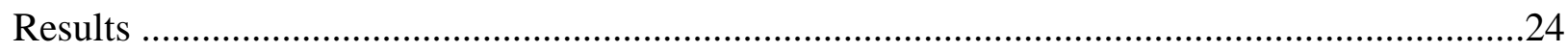

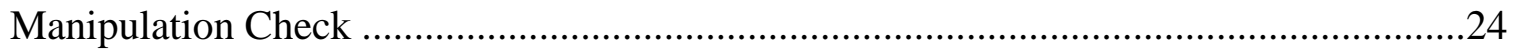

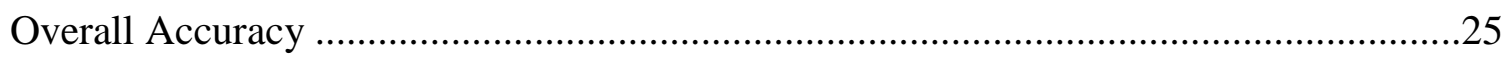

Accuracy as a Function of Context Type and Timing.............................................27

Decisions as a Function of Context Type and Timing...............................................28

Minutia as a Function of Context Type and Timing............................................... 31

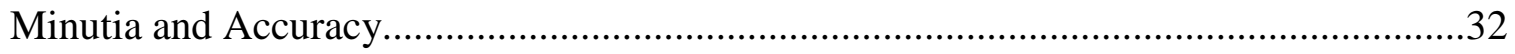

Match-Confidence Composite Score..............................................................33

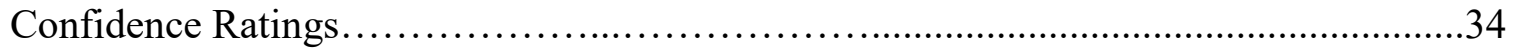




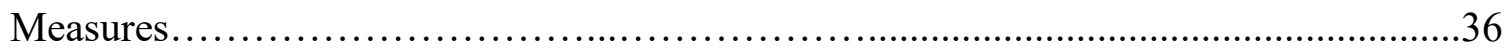

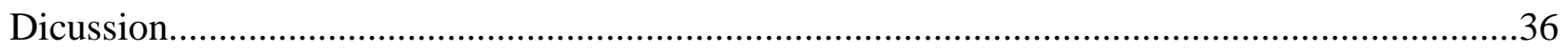

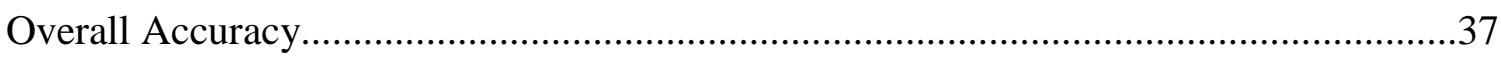

Accuracy, Decisions, and Minutia as a Function of Context.......................................38

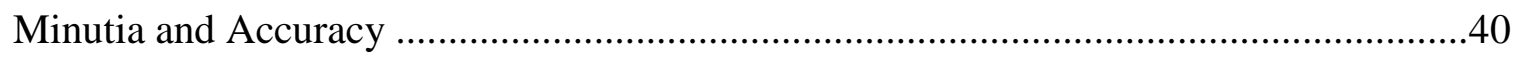

Accuracy and Decisions as a Function of Context Timing............................................40

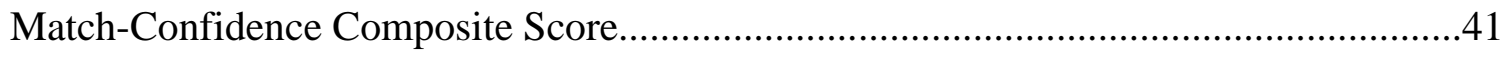

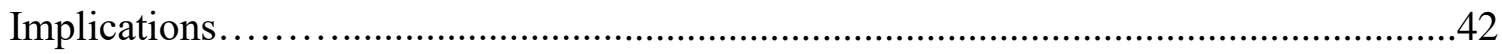

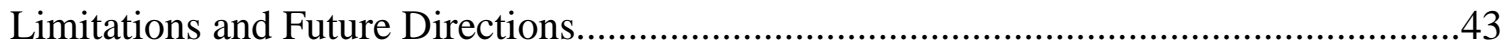

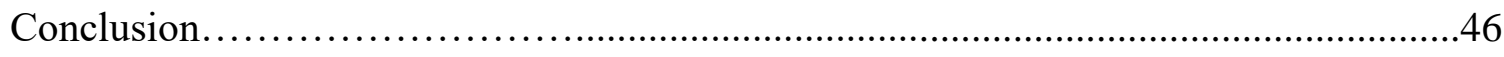

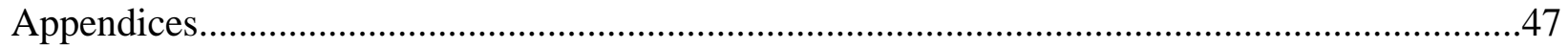

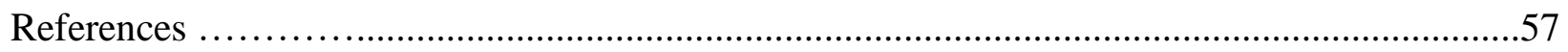




\section{List of Figures}

Figure 1. Participants' accuracy on the nonmatching pair as a function of context timing

Figure 2. Participants' decisions regarding the nonmatching pair as a function of context timing 


\section{List of Appendices}

Appendix A. Demographics Questionnaire

Appendix B. Need for Cognition Scale

Appendix C. Gudjonsson's Compliance Scale

Appendix D. Informed Consent Form

Appendix E. Dependant Measures

Appendix F. Debriefing Form

60 
Evaluating Context Effects on Forensic Examiners: Potential Influences on the Perception of Evidence and Examiners' Decision-Making

Wrongful convictions represent a fundamental error in the justice system and its procedures. When innocent people are convicted of crimes, not only do they waste years of their lives in prison but real perpetrators remain free, putting the rest of the community at risk. In an examination of 194 U.S. cases in which individuals were exonerated due to DNA evidence, the actual perpetrators were found in less than half of all cases (Hampikian, West, \& Akselrod, 2011). During the period in which these innocent individuals were in prison, for a cumulative 2,535 years, some of the actual perpetrators continued to commit crimes. Eventually, 37/77 were ultimately convicted for other violent crimes including rape, murder, and other violent acts. Considering the relative rarity of the actual perpetrator being found, and the evidence of other crimes the 37 perpetrators committed in the absence of conviction, it is likely that the total number of crimes that continued to occur, while an innocent person went to prison, is potentially large.

The Innocence Network, created in 2005, consists of 69 organizations located around the world all with the purpose of proving the innocence of wrongfully convicted individuals. The first organization to free innocent individuals, Centurion Ministries, was founded in 1983 in Princeton, New Jersey (Centurion: Our Mission, 2016). Since its inception, this organization has helped free 54 men and women who were on Death Row. The Innocence Project, founded in 1992, is the largest Innocence Network organization and to date is responsible for 354 exonerations (Innocence Project, 2018). Not only does the Innocence Project work to exonerate innocent prisoners, but their team also works toward reforming the justice system to prevent future wrongful convictions. 
The Innocence Project has identified key contributors to the conviction of these innocent individuals (Innocence Project, 2018). For instance, inadequate defence involves negligence, fraud, or misconduct by prosecutors, police officers, or defence attorneys leading to inadequate defence of the defendant (Innocence Project, 2018). This could include calling an insufficient number of witnesses, not challenging a confession, or concealing evidence supporting the innocence of the defendant (Alpert, 1979). The Innocence Network (2018) has identified at least 13 cases that involve wrongful convictions based on inadequate defence. They have argued for the inclusion of safeguards, such as proper documentation of evidence and quality assurance protocols to help protect against this form of injustice (Innocence Project, 2018).

False confessions or admissions is another factor identified and is estimated to have played a role in the wrongful conviction of more than 1 in 4 DNA exonerees (Innocence Project, 2018). Often in police interrogations, officers disclose specific details about the crime, the crime scene, and the evidence found - specifically, details not shared with the public. Later when obtaining a confession, innocent suspects often repeat the details told to them by interrogators. In most cases, interrogations are only videotaped at the end of the session during the confession, exhibiting the suspect revealing case facts that jurors often think only the perpetrator would know (Kassin et al., 2010). Reforms such as the mandatory recording of all police interrogations have been shown to decrease the occurrence of false confessions (Leo, 2009).

Of primary concern to the current research, misapplication of forensic science was the second most common factor found in the evaluation of DNA exonerees' wrongful convictions, contributing to nearly half (46\%) of cases. Recently, the Innocence Project has revised the title of 'unreliable or invalid forensic science' to 'misapplication of forensic science,' now including sub-categories of unreliable or invalid forensic discipline, insufficient validation of a method, 
misleading testimony, mistakes, and misconduct. Garrett and Neufeld (2009) examined forensic science testimony used in the first 220 DNA exoneration cases and found multiple issues, such as the exclusion of exculpatory evidence, overstating the strength of evidence, and stating statistical and non-statistical information without empirical support.

One example of an unreliable and invalid forensic discipline, also demonstrating insufficient validation for a method, is bite mark comparison. The basic assumption of this type of forensic science is that every individual's bite mark is unique; however, empirical evidence does not exist to support this claim (Garrett \& Neufeld, 2009). Regardless, this analysis is still presented in court as incriminating evidence. In addition to this, bite mark comparison is often subject to misleading court testimony. Because it has not been empirically explored in the population, probabilities that this type of evidence came from a certain individual cannot be validly discussed. However, in court cases of wrongful convictions, analysts have used terms as strong as "identical" and "match," whereas the strongest statements they should be using, e, based on the science, are "consistent with" or "could have come from".

In 2005, the United States Congress authorized the National Academy of Sciences (2009) to conduct a comprehensive study of forensic science. This legislation was passed in response to a call for help from professionals in the forensic science community (Edwards \& Gotsonis, 2009). The investigating committee included a team of experts in various areas, including forensic science, law, and other fields of science and engineering. A 350-page official report was released in 2009 outlining their extensive research and suggested reforms (National Academy of Sciences, 2009). In this report, the committee recognized a need for organizational structures, uniform practices, and better training. The report also noted that many agencies failed to identify the reliability of numerous forensic science methodologies and that it was clear that 
Congress "must take aggressive action" if forensic science's primary issues were to be solved (p. 20). Most notably, they specifically concluded that the development of forensic science research programs could benefit from the findings of cognitive psychology regarding the potential for bias and error in human observers. This demonstrates the need for psychological research concerning the potential for bias in forensic examiners and, therefore, warrants further investigation.

\section{Confirmation Bias}

The idea that contextual information can have an influence on a person's interpretation of stimuli has been extensively researched. Since the early 1900s, psychologists have been exploring the impact of one's knowledge, beliefs, and motivations on a person's perception of visual stimuli, also known as top-down processing (Kassin et al., 2013a). Gottschaldt (1926) explored how past experience influenced the perception of forms. Braly (1933) added to this research by exploring how the presentation of certain figures can influence the perception of subsequent figures. In the early 1940s, researchers now known as New Look theorists classified this way of understanding perception; they considered one's mental set as having an influence on the perception of stimuli in one's environment and considered a person's perception of the real world as not necessarily being what is in the external environment (Bruner and Postman, 1949). Bruner and Postman, the first two New Look theorists, added to the empirical research in the area, specifically demonstrating that perception was shaped by one's expectations. This process is now described as 'confirmation bias'.

Confirmation bias can be defined as a tendency to perceive and seek information that confirms one's existing beliefs (Kassin, Fein, Markus, \& Burke, 2013) and is commonly found in everyday activities. For example, this could include a baseball coach who continues to use strategies that haven proven ineffective, a trial lawyer who always selects juries according to 
false stereotypes, or a mother who believes her son is innocent of shoplifting regardless of seeing him on videotape (Kassin et al., 2013b). In each of these cases, we see what we expect or want to see.

Such processes are often studied in more controlled laboratory conditions. In a series of five studies by Balcetis and Dunning (2006), perception of ambiguous figures was explored as a result of different motivations. Participants were told they would view ambiguous figures (such as a figure that could be interpreted as the letter B or the number 13), and depending on what they perceived, they would be given different tasks. These tasks included a favourable task (like drinking orange juice) or an unfavourable task (like drinking a foul-smelling vegetable smoothie). After manipulating the procedures to control for participants reporting figures in accordance of favourable outcomes, in all five studies, participants consistently perceived the ambiguous figures as the ones they wished to see.

Balcetis and Dunning (2010) later conduced five additional experiments, this time exploring people's estimations of physical distance. Belcetis and Dunning (2010) manipulated the types of desired objects presented to participants across the five studies, including items serving physiological need (water to quench thirst) and socially valued objects (like money and gift cards). In all five studies, participants estimated the distance to the desired object as closer than it actually was. For instance, participants placed themselves further away from chocolate than to feces because they felt they were closer to the chocolate.

Many other studies have supported the existence of confirmation bias, demonstrating the many ways in which the interpretation of stimuli can be skewed as a response to one's desires, expectations, and knowledge. Darley and Gross (1983) asked participants to estimate the academic potential of a young student after inducing high or low expectations. Those who were 
told the student came from a highly-educated family gave more optimistic expectations of the student's potential. Bressan and Dal Martello (2002) demonstrated that when participants were led to believe an adult-child pair were related, participants rated them as looking more similar to each other, even though there was no relation. As is evident, confirmation bias is an overarching type of bias that has the power to impact the interpretation and perception of many types of stimuli regardless of setting.

\section{Forensic Confirmation Bias}

Of particular relevance to psychology and law researchers is forensic confirmation bias, which describes the effect of an individual's knowledge and beliefs influencing the evaluation and interpretation of forensic evidence (Kassin et al., 2013a). Lange, Thomas, Dana, and Dawes (2011) explored how one's expectations influence the interpretation of degraded auditory speech recordings, a stimulus often used as key evidence in court. Before listening to the audio clips, participants were first presented with one of three explanations of what they were about to hear; that the audio clips were drawn from interviews with criminal suspects, that they were from interviews with job candidates, or they were not given any information at all. It was found that participants who believed that the audio clips involved criminal suspects were 4.56 times more likely and 4.15 times more likely to misinterpret the degraded recording than participants who were not presented with context and those who believed they were of a job interview,

respectively. In Experiment 2, following a similar procedure as Experiment 1, results were replicated; this time, participants who believed the recording involved criminal suspects were 7.23 times more likely than those not presented with context to misinterpret the statements.

Importantly, it was found that expectations induced by telling participants the subject matter of the following transcripts created insensitivity to the level of degradation in the audio 
recordings. That is, regardless of the level of degradation of the recording, participants demonstrated a great amount of confidence that they heard the content clearly and correctly (regardless of this being accurate or not). The impact of providing subtle contextual information, like telling participants that audio recordings involve criminal suspects, demonstrates the ease with which audio evidence can be misinterpreted by jurors in court, having the power to transform non-incriminating speech to incriminating. Though this study used only subtle contextual information, jurors in actual criminal trials are provided with much more, creating more expectations about the auditory evidence and potentially an even stronger biasing effect.

Charman, Gregory, and Carlucci (2009) investigated the biases present when evaluating the similarity of facial composites and suspects. Participants were presented with a lineup consisting of four men with similar physical attributes and a facial composite resembling those in the lineup and tasked to make similarity judgements between the composite and each person. In addition, participants were told either that one suspect was identified by two eyewitnesses, or that no identifications were made. Participants who were told of the eyewitness identifications rated the similarity of those suspects as significantly more similar to the facial composite than the other lineup members (Charman et al., 2009). Furthermore, these participants also claimed that the suspect "stood out more" in the lineup (p. 6). However, if participants were later told that another lineup member was identified, their similarity ratings of the previous individual significantly decreased, demonstrating the influence of this suggestibility.

Such findings as those described above demonstrate that one's prior knowledge, specifically concerning contextual information can have an impact on conclusions made about evidence (Charman et al., 2009). These findings also exemplify the power of eyewitness identifications and corroborating evidence in general. The eyewitness identification in this study 
influenced participants' beliefs in guilt and thus influenced the perception of the evidence at hand, namely the facial composite. Thus, one type of evidence not only influences beliefs of guilt but also influences the strength of other pieces of evidence in a case, which can create an exacerbating belief in guilt.

Other studies have found similar examples of bias within a criminal justice setting. Zapf, Kukucka, Kassin, and Dror (2018) found that while forensic mental health evaluators expressed concerns of bias in general, they also had a false belief that one's own willpower is enough to correct for this bias within evaluations. Greathouse and Kovera (2009) found that double-blind administration of lineups (when both the eyewitness and the administrators did not know who the suspect was) led to fewer false identifications than single-blind administrations (when the administrator knew who the suspect was) due to the unconscious biases toward that individual they portrayed. Kassin, Goldstein, and Savitsky (2003) found that interrogators who were given prior information suggesting that the suspect was guilty asked more guilt-presumptive questions, used more interrogation techniques, and exerted more pressure to obtain a confession. Evidently, biases are present in all types of human activity and can be demonstrated within the fields of forensics and law.

\section{Forensic Confirmation Bias and Forensic Science}

These studies demonstrate that forensic confirmation bias can affect all areas of criminal justice. Of particular interest is that which affects the examination of forensic science evidence. This involves the examination of evidence found at a crime scene such as fingerprints, handwriting samples, and DNA against that of a suspect. William Hagan (1894) was among the first scholars to acknowledge the potential for cognitive bias and error in human observers of forensic science, due to the contextual information often provided to forensic examiners before 
analyzing evidence. Though he did not conduct research in this area, he noted that contextual information had the potential to influence the analysis of evidence, impacting the decisions of even the highest experts in the field. Following his observations, he suggested that any contextual information about the case be withheld from examiners.

Forensic examiners, whose jobs are to examine and draw conclusions from forensic evidence, may not always approach evidence objectively (Kassin et al., 2013a). When evidence is submitted to forensic examiners, transmittal letters are often included, which may provide extraneous case information not necessary to the evaluation process (Saks, Risinger, Rosenthal, \& Thompson, 2003). This information may specifically note that the person requesting the evaluation is trying to inculpate (or exculpate) the suspect (Kassin et al., 2013a; Saks et al., 2003). If the examiner does not make a decision consistent with this expected result, investigators often request that the evidence be re-examined (Saks et al., 2003).

Forensic examiners can be exposed to unnecessary contextual information by other means of communication, such as in person, or by phone, when the investigator unintentionally conveys what they are hoping to find (Saks et al., 2003). In one case were investigators wanted lab confirmation that a particular pistol was the murder weapon, an investigator said to the examiner "We know this guy shot the victim and this is the gun he used; all we want you to do is confirm what we already know so we can get the scumbag off the street. We will wait. How quick can you do it?” (Hodge, 1988). This language not only leaves the examiner with knowledge and beliefs regarding the suspect's guilt but also potentially creates a motivation for the examiner to conclude what the officer desires and to compromise the examination. These errors can be compounded given the criteria required for two pieces of evidence to be considered "sufficiently similar" are not clearly and explicitly outlined (Kassin et al., 2013a). That is, if the 
examiner is expecting to find evidence of guilt, they may look for fewer comparison points to match, thus lowering the threshold for guilt. This further illustrates how even seemingly objective evidence can involve subjective judgements.

The first laboratory study examining the effect of context on forensic examiners was conducted by Larry Miller (1984), who based his preliminary work on the extensive findings of confirmation bias in the cognitive and social-psychological literature. To study this, he asked 12 college participants to examine handwriting evidence from a fictitious criminal case; their task was to determine whether the handwriting of the suspect matched that of a sample. They were divided into two groups of six; Group 1 received typical case information (e.g. a synopsis of facts regarding the criminal investigation) while Group 2 received no case information. Importantly, the suspect handwriting samples and the known samples from the crime scene were not authored by the same individual, making any match conclusion erroneous. He hypothesized that the examiners in his study would be influenced by the extraneous case information provided to them by the 'hiring body' (e.g. investigators) implying the suspect's guilt. Four of the six participants in the case information group concluded there was a match between the known sample and the suspect's handwriting, while every participant in the no case information condition correctly concluded they did not match. Miller (1984; 1987) concluded that police officers and attorneys requesting evidence examination should not be given any extraneous case information to better ensure a fair process.

Several other studies demonstrate the myriad ways that bias can subtly creep in to this 'objective' evaluation process. Dror, Peron, Hind, and Charlton (2005) found that forensic examiners' conclusions were more likely to conclude there was a match between fingerprint pairs when they were emotionally aroused (i.e., a particularly heinous crime), and when - as 
above - they were led to believe that the suspect was guilty before the examination. Similarly, Dror and Hampikian (2011) found that examiners evaluating DNA evidence were more likely to find a match in high-profile cases, which often involve a great deal of media attention, and pressure to find the culprit. Dror, Wertheim, Fraser-Mackenzie, and Walajtys (2012) examined the use of Automated Fingerprint Identification Systems (AFIS) which present examiners with a ranked set of fingerprints for comparison, beginning with the most probable match. Dror and colleagues found that when matching fingerprints were presented lower on the list, examiners were more likely to wrongly exclude the matching fingerprint, usually due to spending less time on the examination because these fingerprints are supposedly less likely to be a match.

In a particularly astounding study, Dror, Charlton, and Péron (2006) presented five fingerprint experts with fingerprints they had previously (within the past five years) determined as a match pair. These examiners agreed to be tested within the following 12 months without their knowledge. After verifying that all five pairs were indeed matches with two other expert examiners, the five participant examiners were presented with the same fingerprints but this time with different contextual information. Each examiner was told that the fingerprints were from a high-profile wrongful conviction case involving a pair of fingerprints erroneously identified as a match and that they were to confirm this exclusion. Importantly, examiners were also told to ignore any contextual information when doing their examination. Results indicated that four of the five examiners contradicted their original match finding. This study in particular demonstrates the power of contextual information on the subjective examination of evidence.

After defining the concept of forensic confirmation bias, Kukucka and Kassin (2014) conducted a two-part study investigating the influence of informing examiners of corroborating evidence (specifically, the presence of a confession). In experiment 1, 171 online participants 
were tasked with examining handwriting evidence. All participants were told that the crime was a bank robbery, that the perpetrator left a note, and that a suspect was apprehended for a 3-hour questioning. The only difference between the two groups was that one group was told that the suspect confessed after the 3-hour questioning but later recanted the confession, saying it was coerced, while the other group was not told of a confession. Those in the confession-present condition were significantly more likely to determine a match between the handwriting evidence and that of the suspect than those in the confession-absent condition (Kukucka \& Kassin, 2014). A match-confidence composite score was also created, merging match judgements and the participants' self-rated confidence in their decision. It was found that participants in the confession-present condition had significantly higher composite scores than those in the confession-absent condition. In line with Experiment 1, in Experiment 2, which included two time points, participants who were presented with contextual information at time 2 were more likely to judge the handwriting pair as a match than they were at time $1(35.71 \%$ versus $14.29 \%$, respectively).

Other studies have come to similar conclusions. For example, Kassin, Bogart, and Kerner (2012) conducted an archival analysis of Innocence Project DNA exonerations to examine the theory that one type of evidence against a suspect can affect the pursuit and interpretation of other supporting evidence. It was found that when a confession was present, errors in other types of evidence were significantly more likely to exist in that case; the leading evidence error being invalid or improper forensic science. Dror et al. (2011) examined the consistency of forensic examiners' conclusions and the impact of contextual information on their analyses. It was found that not only did the presentation of contextual information affect their conclusions, but it also changed the way in which the experts did their analyses (e.g., affecting 
the analysis of the latent crime scene fingerprint specifically). Dror (2013) also noted that experts in particular are more susceptible to cognitive biases and the influence of contextual information due to the method of examining becoming more based on short-cuts and relying on past experience.

A recent study sought to explore the opinions of forensic examiners regarding the potential for bias within their own examinations (Kukucka, Kassin, Zapf, \& Dror, 2017). The sample included over 400 experienced forensic examiners from 21 different countries accessed via email and were asked about their experiences and opinions. It was found that there was an overwhelming denial of bias within their own work. Most examiners described their examinations and conclusions as nearly faultless and that they were not susceptible to bias. They also demonstrated a lack of understanding and acceptance of this cognitive bias and fewer than half supported changes to the traditional procedure to incorporate a method shown to decrease the presence of this bias. Lastly, they demonstrated a bias blind spot, acknowledging bias in other domains but not in their own or even in other examiners but not in themselves.

Charlton, Fraser-Mackenzie, and Dror (2010) interviewed 13 experienced fingerprint examiners from a variety of law enforcement agencies to investigate the emotional and motivational factors associated with performing these types of examinations. This analysis revealed some key factors relating to their job satisfaction, including feelings of satisfaction associated with catching criminals and positive emotions when finding a match between crime scene evidence and that of a suspect. Therefore, not only are examiners influenced by contextual information, but they may also be influenced by their own motivations (Charlton et al., 2010). Furthermore, they found experts to have a need for cognitive closure (i.e., the psychological need to avoid unresolved issues and conclude the case investigation). This need can affect an 
examiner's interpretation when additional supporting evidence is needed in an investigation (Charlton et al., 2010).

Stoel, Dror, and Miller published a commentary regarding their research published in 1984. The 2014 paper noted that despite 30 years in between their original research and their current paper, there was still a need for procedural changes. The purpose of the article was to bring the 1984 study to the attention of the forensic community, reiterating the issues found regarding forensic confirmation bias and noting that procedural changes have yet to take place in forensic evidence examination (Stoel et al., 2014). However, the studies conducted until now have only started to identify the psychological processes involved in this phenomenon, requiring much additional research investigating the way in which contextual information affects the examination of evidence (Dror \& Cole, 2010).

Kassin and colleagues (2013a) noted that the potential for the examinations and subjective judgements to vary in multiple ways creates a need for much additional research in the area. Furthermore, due to the lack of reform having taken place, additional research regarding the presence of forensic confirmation bias and how this bias operates can act to further strengthen the notion that a change in forensic examining procedure is necessary (Stoel et al., 2014). Most notably, the report published by the National Academy of Sciences (2009) highlighted the scarcity of research pertaining to cognitive biases within forensic science and stated the need for an expansion of this research in the future.

\section{The Current Study}

The above research effectively demonstrates the impact that cognitive biases have on people's experiences with objects in their environment. More specifically, the impact of confirmation bias has been demonstrated in a variety of ways, even affecting such things as the 
physical distance people create between themselves and a desired or undesired object (Balcetis \& Dunning, 2010). Confirmation bias is a type of human error and, as such, can be applied to any type of work by human observers. However, it was only recently applied to the area of forensic examinations. Though forensic confirmation bias is a new concept, multiple studies exist that demonstrate that top-down processes, or a person's knowledge, beliefs, and motivations, affect examiners' interpretations of forensic evidence.

Because research surrounding forensic confirmation bias is still in its early stages, there is a lot we have yet to learn. Though there is plenty of research demonstrating its existence, no research has yet looked at the way in which this bias operates. Currently, we understand that when forensic examiners are presented with contextual information before analysis, the results of the analysis are often different or influenced by this information. The effect of this information (forensic confirmation bias) is taking place during the actual examination. The effect of this bias needs to be examined in more detail to be able to understand what happens between the presentation of contextual information and the affected conclusion from the examiner.

For instance, as previous research has demonstrated, some amount of ambiguity in evidence is necessary for contextual information to have an effect on examiners. In other words, if fingerprint samples look completely different, knowing that the suspect confessed to the crime is unlikely to affect an examiner's final conclusion about making a nonmatch judgement. However, if it is difficult to determine if the two fingerprints originated from the same source, for instance because the latent fingerprint retrieved from the crime scene is extremely distorted, then contextual information is substantially more likely to influence the examiner's final conclusion. Though we know this, we do not know if the information is affecting the examiner's perception of the visual stimuli or if the ambiguous stimuli lead to the examiner having to make 
a more subjective judgement about the evidence and that the contextual information influences the decision-making phase.

We can reason that the presentation of contextual information is either affecting the examiner's perception or his/her decision-making. The perception of the evidence is the examiner doing the visual examination of the evidence. This is followed by the examiner's contemplation about his/her perception and making the decision of whether the two pieces of evidence (e.g., fingerprints) are similar enough to conclude a match. What is yet to determine is whether the contextual information is relevant to the perception of the evidence, the examiner's decision-making process, or both. If we know whether this bias is affecting the perception or decision-making, this information will allow us to properly inform our suggestions of reform in forensic examination procedure. This information will tell us exactly what part of the examination must be of focus in the procedure reform.

In addition to this, statistical models are now being introduced to help with the objectivity of fingerprint evidence examination; however, they only aid in the final conclusions about the forensic evidence. Human examiners perceive the evidence (i.e., a latent fingerprint from a crime scene and fingerprints from a suspect) and put their findings into the model (i.e., the number of minutia or points of reference in each fingerprint). The model will then take this information and make a conclusion about the fingerprints. This eliminates the examiner's decision-making and the potential for contextual information to influence this process; however, it does not account for contextual information influencing an examiner's perception. Therefore, if we demonstrate that contextual information is in fact affecting examiners' perception of evidence, then we will also demonstrate that this technological advancement cannot be used as a solution for this problem. 
The current study intended to fill this gap in research. This study evaluated whether contextual information affects an examiner's perception of fingerprint evidence or his/her decision-making process regarding whether the fingerprints are a match, nonmatch, or if it is inconclusive. To evaluate this, university students at Ryerson University were trained in fingerprint examination and subsequently examined four pairs of fingerprints. Two of three groups were exposed to contextual information about the suspect (either implying innocence or guilt), but to inspect what part of the examination is affected by this information, the context was presented at two different stages. One group was presented with the information prior to the examination of any fingerprints and one group was presented with the information after the examination of fingerprints but before comparison.

To investigate whether context affected perception and/or decision-making, we first looked at any differences between those who were presented with contextual information and those who were not. This involved looking at differences between the groups presented with contextual information (before or after fingerprint examination) versus the group not presented with contextual information. Secondly, we looked at our main research question, identifying any differences between the groups presented with contextual information at different times to inspect whether the contextual information is affecting perception, decision-making, or both. After this, we inspected differences between those who were presented with contextual information implying innocence versus that implying guilt. Based upon the presented research in cognitive psychology regarding the impact of top down processes on perception and that in forensic psychology regarding the effect of one's knowledge on his/her conclusions about evidence, we hypothesized that the contextual information would affect both the examiner's perception of the evidence as well as their decision-making process. 
Also based upon the research of Kukucka (2014) finding the confession condition (representing context implying guilt) to lead to more match conclusions than either the alibi or denial conditions (representing context implying innocence), we hypothesized that those presented with contextual information implying guilt would be more likely to conclude there is a match between fingerprints. Kukucka and Kassin (2014) created a match-confidence composite score combining participants' match judgements with their associated confidence in their decision. They found that participants in the confession condition (representing context implying guilt) had significantly higher match-confidence composite scores (Kukucka and Kassin, 2014). Due to this finding, we created match-confidence composite scores as well and hypothesized that higher scores would be associated with those who were presented with contextual information implying the suspect's guilt.

\section{Method}

\section{Participants}

A power analysis, using $\mathrm{G}^{*}$ Power software, was conducted to determine the number of participants required to obtain $80 \%$ power for a mixed analysis of variance with a withinbetween interaction when the effect size is moderate and with 6 conditions and 3 dependant measures. Results indicated that 135 participants would be needed for sufficient power. After the exclusion of the first 16 participants due to a slight change in procedure and enhancement of contextual information, the final sample consisted of 129 (83.7\% women) undergraduate students enrolled in an Introductory Psychology course, recruited through the Sona online recruitments system. Ages ranged from 17 to 56, with a mean of $20.47(S D=5.56)$. Our sample was predominantly White (45.0\%), with 23 (17.8\%) identifying as South Asian, 11 (8.5\%) identifying as other, and the remaining participants identifying as Black, Filipino, Chinese, West 
Asian, Latin American, Aboriginal, Arab, and some identifying as more than one ethnicity (see Appendix A). All participants were compensated 1\% toward their Introduction to Psychology course grade for their participation.

\section{Design}

The current study is a 2 (context presentation: before observation vs. after observation) by 2 (context direction: implying innocence vs. implying guilt) design. Additionally, as a baseline, there is also a no context condition, totaling to five possible conditions. Therefore, participants could be presented with contextual information implying guilt, before observation; implying guilt, after observation; implying innocence, before observation; implying innocence, after observation; or no context. Participants were first trained in fingerprint examination by watching three training videos and subsequently examined two pairs of fingerprints (one a nonmatching pair and one a matching pair) from independent cases.

\section{Materials}

Videos. Three videos, created by Christopher Birch and found on Youtube.com were used for the training portion of the study; they demonstrated fingerprints, fingerprint examination, and how to properly analyze fingerprints to determine a match, nonmatch, or inconclusive result. These videos combined to almost 10 minutes in length and participants were instructed to pause and/or rewind the videos as they wished. The first video described the three main classes of fingerprints, the second video described the subclasses of fingerprints, and the third video defined and described minutia and summarized all three videos. The last video stated that 8-12 points of reference, or minutia, should be used as a guide for making a match judgement, which is typically the accepted range in real examinations. These videos are freely accessible to the public on Youtube.com (https://www.youtube.com/watch?v=8f5kfcSdN9c; 
https://www.youtube.com/watch?v=djBIgQTgruc; https://www.youtube.com/watch?v=jWCyu2KmDw).

Fingerprints. Fingerprints were obtained from www.nist.gov, a database available from National Institute of Standards and Technology (NIST). The NIST Biometric Image Software was developed for the Federal Bureau of Investigation (FBI) and Department of Homeland Security (DHS). This software was created solely by employees of the U.S. Government and is freely distributed and considered public domain. Two pairs of fingerprints were presented to participants; Pair A included two different fingerprints downloaded from the above database and comprised the non-matching pair. Pair B included one high-quality fingerprint downloaded from the above database, and the second fingerprint being an altered, lower quality of the same fingerprint, thus comprising the matching pair.

Need for Cognition Scale. Participants completed the abbreviated version of the Need for Cognition scale (Cacioppo, Petty, \& Kao, 1984) adapted from the original 34 item scale (Cacioppo \& Petty, 1982). This scale assesses participants' enjoyment of engaging in thinking and effortful cognitive activity (Cacioppo et al., 1984). The abbreviated version consists of 18 items to which individuals indicate how much they agree or disagree with statements on a 9point Likert-type scale ranging from very strongly disagree to very strongly agree Final scores range from -72 to +72 , with higher scores indicating higher levels of need for cognition. In the original paper, this scale possessed a reliability coefficient of .87. In the current sample, the scale produced a Cronbach's alpha of .89, demonstrating a high level of internal consistency. See Appendix B.

Gudjonsson's Compliance scale. Participants also completed the Gudjonsson Compliance scale. This scale includes 20 true-false statements which assess how susceptible the 
participant is to suggestibility and compliant behaviour (Gudjonsson, 1989). Scores range from 0 to 20 with higher scores indicating a higher degree of compliant behaviour In the original paper, this scale possessed a reliability coefficient of .88. In the current sample, the scale produced a Cronbach's alpha of .71, demonstrating an acceptable level of internal consistency. See Appendix C.

\section{Procedure}

Participants were tested in a psychology research testing room at Ryerson University. Written and verbal informed consent was obtained prior to the commencement of the study (see Appendix D). Participants were told that the study's objective was to compare the observations and conclusions of forensic examiners to undergraduates in order to determine if forensic examiners are significantly better at doing these analyses. Thus, participants were told that they would be analyzing fingerprints from real cases in order to directly compare what they found to what the forensic examiners found in the same cases. Participants first underwent training (through the use of the aforementioned videos) teaching them how to find and count the number of minutia present in a fingerprint, how to compare two fingerprints, and how to subsequently come to a conclusion (finding an match, nonmatch, or inconclusive result). Following training, participants examined two pairs of fingerprints, the presentation of which was counterbalanced.

Context direction included the contextual information either suggesting that the suspect was innocent or guilty. In the innocence-presumptive condition, participants were told that it was the defence that hired the forensic examiner and that they strongly believe their defendant is innocent due to the individual not confessing to the crime regardless of being interrogated by police and because an eyewitness that was at the scene of the crime identified a different individual in the lineup. In the guilt-presumptive condition, participants were told that it was the 
prosecution that hired the forensic examiner and that they strongly believe the suspect is guilty due to the individual confessing to the crime after a short interrogation and because an eyewitness that was at the scene of the crime identified this individual in the lineup. Gender of the suspect was never specified.

Participants were randomly assigned to one of five conditions involving the type and timing of the context presented. Conditions 1 and 2 involved verbally providing the participants with contextual information about the case before they were presented with any fingerprints. This information suggested that the suspect was guilty (condition 1) or that the suspect was innocent (condition 2). After being told this contextual information, participants were provided with the latent crime scene fingerprint, asked to count and record the number of minutia and their confidence in their number, then were presented with the suspect fingerprint and again were asked to count and record the number of minutia and their confidence in their number. They then compared both fingerprints and made a final conclusion. Those in conditions 3 and 4 were first provided with the latent crime scene fingerprint, asked to count and record the number of minutia and their confidence in their number, then were presented with the suspect fingerprint and again were asked to count and record the number of minutia and their confidence in their number. After these numbers were submitted, participants were told the contextual information (that implying guilt in condition 3 or innocence in condition 4). Participants were then asked to compare the two fingerprints and make a final conclusion.

For condition 5, participants were first provided with the latent crime scene fingerprint, asked to count and record the number of minutia and their confidence in their number, then were presented with the suspect fingerprint and again were asked to count and record the number of minutia and their confidence in their number. They then compared both fingerprints and made a 
final conclusion, therefore not being presented with contextual information. All participants were in the same condition for both fingerprint pairs. For instance, if a participant was presented with contextual information implying guilt before the presentation of the first set of fingerprints, this was also the case for the second set.

Dependant measures included the numbers of minutia observed in both the latent and suspect fingerprints (continuous variables), the participant's final conclusion (match, non-match, or inconclusive), and their confidence ratings regarding these decisions on a 7-point Likert scale (see Appendix E). Participants first provided the number of minutia they counted in the latent fingerprint, followed by their confidence that they counted the correct number. Secondly, participants provided the number of minutia they counted in the suspect fingerprint, followed by their confidence that they counted the correct number in that fingerprint. Finally, participants circled their final judgement about the two fingerprints (match, nonmatch, or inconclusive), followed by their confidence in their decision (for decisions of match or nonmatch only).

Participants worked on one case at a time, analyzing the evidence and submitting the numbers of minutia, their final conclusion, and their confidence ratings before moving on to the next pair of fingerprints. After all fingerprints were analyzed, participants completed the Need for Cognition Scale (Cacioppo et al., 1984) and the Gudjonsson's Compliance scale (Gudjonsson, 1989) as well as a demographics questionnaire. When participants completed all tasks, they were debriefed about the true nature of the study and thanked for their participation (see Appendix F). 


\section{Results}

\section{Manipulation Check}

After collecting data from 16 participants, Chi-square tests of independence were conducted to investigate whether the contextual information manipulation was influencing participants' decisions. Originally, context simply included a sentence stating "In this case, the suspect confessed to the crime after an interrogation and the suspect was identified by an eyewitness" in the guilty condition and "In this case, the suspect did not confess to the crime after an interrogation and a different individual was identified by an eyewitness" in the innocent condition. No differences were found in decisions between participants given context and those who were not. After this preliminary finding, we suspected that participants were not attending to the information in these statements, and so contextual information was enhanced for all remaining participants.

Participants now either were told "In this case, it was the prosecution that hired the forensic examiner. They strongly believe the suspect is guilty due to the suspect confessing to the crime and an eyewitness that was at the scene of the crime identified this individual in the lineup" (guilty condition), or "In this case, it was the defence that hired the forensic examiner. They strongly believe their defendant is innocent; this individual did not confess to the crime regardless of being interrogated by police and an eyewitness that was at the scene of the crime identified a different individual in the lineup" (innocent condition). In addition, directly after viewing the training videos, participants were given more explicit information about how forensic examiners are typically hired by either the prosecution or defence, which could influence their decisions (that is, they may lack neutrality). 


\section{Overall Accuracy}

To assess whether there were differences associated with the difficulty of assessing each pair, we first looked at the frequencies of accurate judgements. Accurate judgements consist of participants making a match judgement when presented with the matching fingerprint pair and a nonmatch judgement when presented with the nonmatching fingerprint pair. Variables were created representing the overall accuracy of the matching fingerprint pair and the nonmatching fingerprint pair. First, variables were created representing the accuracy of participants' judgements for each set and for each fingerprint pair. That is, four variables were created representing the accuracy of participant's judgements when presented the matching fingerprint pair in set 1 , the nonmatching fingerprint pair in set 1 , the matching fingerprint pair in set 2 , and the nonmatching fingerprint pair in set 2. Each variable was coded as 1, being accurate, and 2, being inaccurate.

Next, these variables were combined to create two variables representing the accuracy of participant's judgements when presented the matching fingerprint pair in both sets 1 and 2, and another representing accuracy when presented the nonmatching fingerprint pair in both sets 1 and 2. Because all participants were presented with the matching and nonmatching fingerprint pairs only once, in either set 1 or 2 , these two variables represented every judgment. Frequencies were then acquired for both these variables, providing the percentage of accurate versus inaccurate judgements for each pair. For the matching fingerprint pair, participants were accurate $71.9 \%$ of the time (92 participants out of 129) and inaccurate $28.1 \%$ of the time (36 participants out of 129). For the nonmatching fingerprint pair, participants were accurate $52.7 \%$ of the time and inaccurate $47.3 \%$ of the time. To compare participants' accuracy to chance, onesample t-tests were conducted using the above accuracy variables. For the matching fingerprint 
pair, participants performed better than chance, $t(127)=-5.48, p<.001, r=.19$. For the nonmatching fingerprint pair, participants performed no better than chance, $t(128)=-0.62, p=$ .540. The percentages were averaged to gain the overall accuracy across fingerprint pairs. Overall, participants were accurate $62.3 \%$ of the time and inaccurate $37.7 \%$ of the time. Following this, two new variables were created from the original four variables representing accuracy, now representing the accuracy of participant's judgements for both the matching or nonmatching fingerprint pair in set 1 , and another representing this accuracy for set 2. This was used to determine if participants did better on one set over the other (e.g., if by the second set they had more practice and were performing better). We found that order of fingerprint pair presentation did not affect the accuracy of judgements. Participants were accurate in their decisions for Set 1 (the pair presented first) $62 \%$ of the time compared to $62.5 \%$ of the time for Set 2 (the pair presented second) regardless of whether they received the match or non-match pair.

\section{Accuracy as a Function of Context Type and Timing}

A 2 (context type: suggesting innocence vs. suggesting guilt) x 2 (context timing: before observation vs. after observation) multivariate analysis of variance on participants' accuracy for both the matching and nonmatching fingerprint pairs. It was hypothesized that contextual information would affect both the examiner's perception of the evidence as well as their decision-making process, and therefore, that it would affect participants' accuracy. Results were only partially in line with this hypothesis. For the matching fingerprint pair, the resulting $2 \times 2$ ANOVA revealed no main effects of context type $F(1,79)=0.42, p=.519$ between those who were accurate $(M=0.55, S D=0.50)$ and inaccurate $(M=0.48, S D=0.51)$, or for context timing, $F(1,79)=0.95, p=.333$ between those who were accurate $(M=0.60, S D=0.50)$ and inaccurate 
$(M=0.48, S D=0.51)$. For the nonmatching fingerprint pair, while there was no main effect of context type $F(1,79)=0.69, p=.409$ between those who were accurate $(M=0.50, S D=0.51)$ and inaccurate $(M=0.58, S D=0.50)$, there was a main effect of context timing $F(1,79)=5.95, p$ $=.017, \eta_{\mathrm{p}}{ }^{2}=.07$, such that participants were more inaccurate when given context after fingerprint observation $(M=0.60, S D=0.50 ; 28$ inaccurate judgements $)$ than when given context before observation $(M=0.33, S D=0.48 ; 12$ inaccurate judgements $)$. Therefore, though the type of context given did not have an effect, the timing in which this context was given did. See Figure 1.

Additionally, due to the multivariate analyses of variance not including the no context conditions, so to create a fully crossed analysis, one-way analyses of variance were conducted assessing the effect of context type and timing on participants' accuracy for both the matching and nonmatching fingerprint pairs. For the matching pair, the resulting ANOVA revealed no main effects of context type, $F(2,127)=0.61, p=.545$, or context timing $F(2,127)=0.89, p=$ .413. Similarly for the nonmatching pair, there were no main effects of context type, $F(2,128)=$ $0.24, p=.789$, or context timing $F(2,128)=2.63, p=.076$.

\section{Decisions as a Function of Context Type and Timing}

Similarly, we ran a 2 (context type: suggesting innocence vs. suggesting guilt) x 2 (context timing: before observation vs. after observation) multivariate analysis of variance on participants' decisions for both the matching and nonmatching fingerprint pairs. Therefore, not whether these decisions were accurate, but whether contextual information influenced the frequency of participants making match, nonmatch, or inconclusive judgements. While it was hypothesized that contextual information would affect participants' decisions, for the matching 
fingerprint pair, the resulting $2 \times 2$ ANOVA revealed no main effects of context type, $F(1,79)=$ $0.17, p=.680$ between those who made a match judgment $(M=0.55, S D=0.50)$, nonmatch judgment $(M=0.40, S D=0.52)$, or inconclusive judgment $(M=0.55, S D=0.52)$.

Similarly, a 2x2 ANOVA revealed no main effects of context timing, $F(1,79)=1.03, p=$ .313 between those who made a match judgment $(M=0.60, S D=0.50)$, nonmatch judgment $(M$ $=0.50, S D=0.53)$, or inconclusive judgment $(M=0.45, S D=0.52)$. For the nonmatching fingerprint pair, there was no main effect of context type $F(1,79)=0.15, p=.702$ between those who made a match judgment $(M=0.58, S D=0.50)$, nonmatch judgment $(M=0.50, S D=0.51)$, or inconclusive judgment $(M=0.57, S D=0.54)$. Similarly, a $2 \times 2$ ANOVA revealed no main effects of context timing $F(1,79)=3.54, p=.063, \eta_{\mathrm{p}}{ }^{2}=.04$ between those who made a match judgment $(M=0.73, S D=0.45)$, nonmatch judgment $(M=0.45, S D=0.50)$, or inconclusive judgment $(M=0.57, S D=0.54)$; however, this main effect was marginal. Therefore, participants' decisions regarding the nonmatching fingerprint pair may have been influenced by when context was presented. Participants made more (incorrect) match decisions when context was presented after observation ( $M=0.57, S D=0.65 ; 24$ match judgements) than when presented before observation ( $M=0.83, S D=0.56 ; 9$ match judgements). See Figure 2. Furthermore, it was hypothesized that those presented with contextual information implying guilt would be more likely to conclude there is a match between fingerprints. Contrary to this prediction, for the matching and nonmatching fingerprint pairs respectively, the decisions of participants presented with context implying guilt $(M=0.41, S D=0.72 ; M=0.72, S D=0.61)$ were similar to those presented with context implying innocence $(M=0.36, S D=0.72 ; M=$ $0.67, S D=0.64)$. 


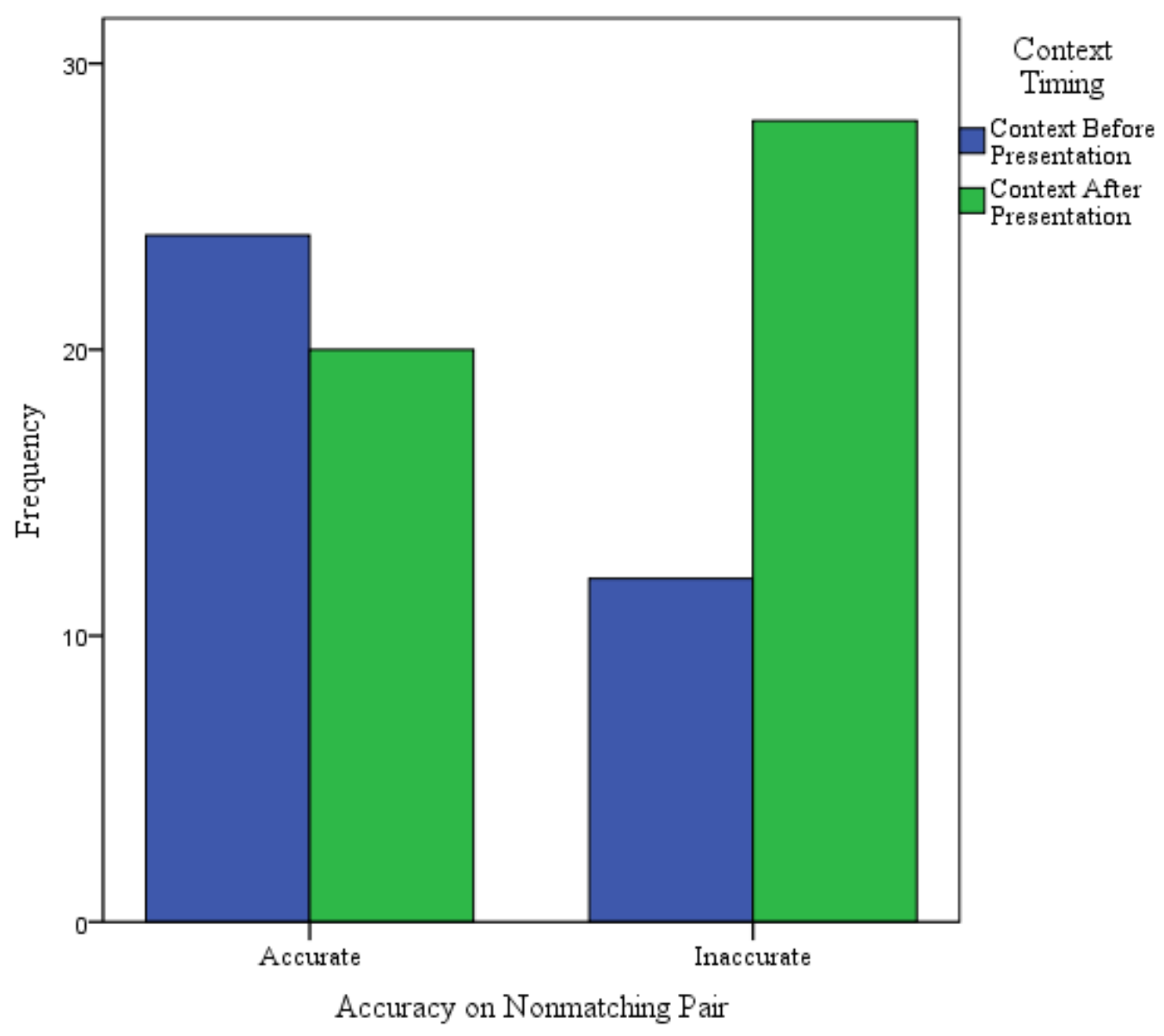

Figure 1. Participants' accuracy on the nonmatching pair as a function of context timing.

Lastly, one-way analyses of variance were conducted including the no context conditions assessing the effect of context type and timing on participants' decisions for both the matching and nonmatching fingerprint pairs. For the matching pair, the resulting ANOVA revealed no main effects of context type, $F(2,127)=0.46, p=.633$, or context timing $F(2,127)=0.86, p=$ 427. Similarly for the nonmatching pair, there were no main effects of context type, $F(2,128)=$ $0.23, p=.799$, or context timing $F(2,128)=1.81, p=.167$. 


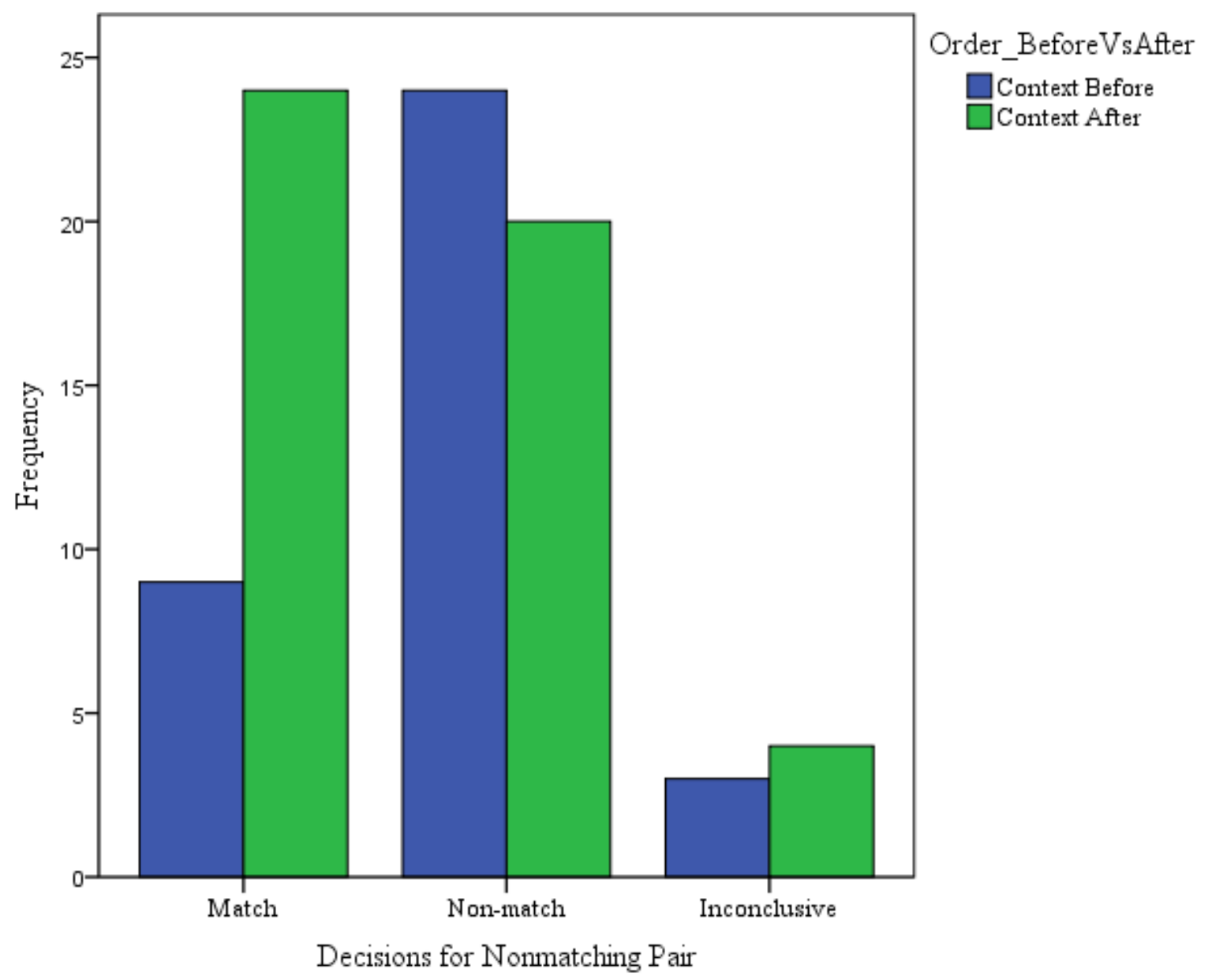

Figure 2. Participants' decisions regarding the nonmatching pair as a function of context timing.

\section{Minutia as a Function of Context Type and Timing}

Participants were asked to count the number of minutia within each fingerprint in both pairs. It is important to note that the accuracy of minutia numbers could not be assessed due to the true number of minutia in each fingerprint being unknown and, of course, open to subjective judgement. One-way analyses of variance were conducted regarding minutia numbers counted in both the matching and nonmatching fingerprint pairs to assess whether the type of contextual information given (context implying guilt, context implying innocence, or not provided any 
context) affected the numbers of minutia counted. While it was hypothesized that context would affect the numbers of minutia counted, no significant differences were found.

Within the matching pair, this was true for the latent print, $F(2,127)=0.52, p=.598$, such that the numbers of minutia counted were similar when given context implying guilt $(M=$ $5.82, S D=3.19)$, innocence $(M=5.84, S D=3.19)$, or no context $(M=6.78, S D=7.29)$. And also for the suspect print, $F(2,127)=1.21, p=.302$, such that the numbers of minutia counted were similar when given context implying guilt $(M=7.90, S D=3.86)$, innocence $(M=7.50, S D$ $=3.99)$, or no context $(M=9.82, S D=11.48)$. Within the nonmatching pair, this was true for the latent print, $F(2,128)=1.17, p=.314$, such that the numbers of minutia counted were similar when given context implying guilt $(M=12.00, S D=6.00)$, innocence $(M=12.20, S D=5.49)$, or no context $(M=14.09, S D=8.99)$. And also the suspect print, $F(2,128)=1.24, p=.293$, such that the numbers of minutia counted were similar when given context implying guilt $(M=10.26$, $S D=5.68)$, innocence $(M=11.00, S D=6.34)$, or no context $(M=12.80, S D=10.06)$.

We ran a 2 (context type: suggesting innocence vs. suggesting guilt) x 2 (context timing: before observation vs. after observation) multivariate analysis of variance on participants' minutia numbers for both the matching and nonmatching fingerprint pairs. For the matching fingerprint pair, the resulting 2x2 ANOVA revealed no main effects of context type on either the latent fingerprint, $F(1,79)=0.04, p=.836$, or the suspect fingerprint, $F(1,79)=0.08, p=.785$. However, there was a main effect of context timing on the latent fingerprint, $F(1,79)=3.80, p=$ $.055, \eta_{\mathrm{p}}{ }^{2}=.05$, but no main effect of context timing on the suspect fingerprint $F(1,79)=2.03, p$ $=.158$. For the latent fingerprint, participants counted more minutia when presented with context before fingerprint observation $(M=6.61, S D=3.31)$ than when presented after observation $(M=5.25, S D=2.97)$. For the nonmatching fingerprint pair, the resulting $2 \times 2$ 
ANOVA revealed no main effects of context type on either the latent fingerprint, $F(1,79)=0.23$, $p=.635$, or the suspect fingerprint, $F(1,79)=0.76, p=.386$. However, there was a main effect of context timing on the latent fingerprint, $F(1,79)=6.86, p=.011, \eta_{\mathrm{p}}^{2}=.08$, but no main effect of context timing on the suspect fingerprint $F(1,79)=3.47, p=.066$, though this main effect was marginal. As with the matching fingerprint pair, for the latent fingerprint, participants counted more minutia when presented with context before fingerprint observation $(M=13.97, S D=5.27)$ than when presented after observation $(M=10.75, S D=5.65)$.

\section{Minutia and Accuracy}

One-way analyses of variance were conducted regarding minutia numbers counted in both the matching and nonmatching fingerprint pairs as a function of the accuracy of participants' judgements. That is, we assessed whether the numbers of minutia participants counted were affected by whether the participants' final judgements concerning that pair of fingerprints (whether it was a matching or nonmatching pair) were accurate or inaccurate. All results demonstrated that participants' accuracy did not influence the numbers of minutia counted. Within the matching pair, this was true for the latent print, $F(1,127)=2.48, p=.118$, and suspect print, $F(1,127)=2.64, p=.107$. Within the nonmatching pair, this was true for the latent print, $F(1,128)=0.12, p=.733$, and suspect print, $F(1,128)=0.16, p=.692$.

\section{Match-Confidence Composite Score}

Using Kukucka's (2014) approach, match-confidence composite scores were created to assess differences as a function of contextual information. To do this, match decisions were coded as +1 , nonmatch decisions were coded as -1 , and inconclusive decisions were coded as 0 . The product of these values and the confidence ratings associated with the decisions were computed into two new variables, for both the pairs presented at set 1 and set 2, producing the 
match-confidence composite score. Subsequently, a one-way analysis of variance was conducted using these computed values and the context variable, assessing any differences as a function of context. It was hypothesized that participants who were presented with contextual information implying the suspect's guilt would have higher composite scores than those in the other context conditions. Contrary to our prediction, for set 1 (the fingerprint pair presented to participants first), match-confidence composite scores did not differ between those presented with either type of context $(M=1.61, S D=4.71)$ and those not presented context $(M=0.89, S D$ $=4.22), F(2,127)=1.62, p=.202$. Similarly, for set 2 (the fingerprint pair presented to participants last), match-confidence composite scores again did not differ between those presented with either type of context $(M=1.16, S D=4.56)$ and those not presented context $(M=$ $0.82, S D=4.64), F(2,127)=0.09, p=.910$.

Furthermore, we ran a 2 (context type: suggesting innocence vs. suggesting guilt) x 2 (context timing: before observation vs. after observation) multivariate analysis of variance on match-confidence composite scores for both fingerprint sets (first and second) examined. For set 1, the resulting $2 \times 2$ ANOVA revealed no main effect of context type, $F(1,79)=3.58, p=.062$, though this effect was marginal. However, there was a main effect of context timing, $F(1,79)=$ $4.06, p=.047, \eta_{\mathrm{p}}^{2}=.05$, such that composite scores were higher when context was presented after fingerprint observation $(M=2.30, S D=4.35)$ than when presented before observation $(M=$ $0.72, S D=5.07)$. These main effects were qualified by a context type $\mathrm{x}$ context timing interaction, $F(1,79)=5.82, p=.018, \eta_{\mathrm{p}}^{2}=.07$. Simple effects revealed that when context was presented before observation, composite scores were higher for those presented with context implying innocence $(M=3.00, S D=4.46)$ than for those presented with context implying guilt $(M=-1.56, S D=4.69), p=.003$, mean difference $=4.56,95 \%$ CI $[1.64,7.47]$. For set 2 , there 
was no main effects of either context type, $F(1,79)=0.16, p=.690$, or context timing, $F(1,79)=$ $1.66, p=.202$. Furthermore, the interaction was not significant, $F(1,79)=0.49, p=.487$.

Similar to above, one-way analyses of variance were conducted assessing the effect of context type and timing including the no context conditions on match-confidence composite scores for both sets 1 and 2. For set 1, the resulting ANOVA revealed no main effect of context type on composite scores, $F(2,127)=1.62, p=.202$. Similarly, for set 2 , the resulting ANOVA revealed no main effect of context type on composite scores, $F(2,127)=0.09, p=.910$.

\section{Confidence Ratings}

We ran a 2 (context type: suggesting innocence vs. suggesting guilt) x 2 (context timing: before observation vs. after observation) multivariate analysis of variance on participants' confidence ratings regarding minutia numbers and decisions for both the matching and nonmatching fingerprint pairs. For the matching fingerprint pair, the resulting $2 \times 2$ ANOVA revealed no main effects of context type on the latent fingerprint between those presented with context implying innocence $(M=3.57, S D=1.44)$ and implying guilt $(M=3.33, S D=1.36)$, $F(1,65)=2.16, p=.146$, the suspect fingerprint between those presented with context implying innocence $(M=4.07, S D=1.37)$ and implying guilt $(M=4.08, S D=1.24), F(1,65)=0.31, p=$ .578 , or decisions between those presented with context implying innocence $(M=5.24, S D=$ $1.22)$ and implying guilt $(M=4.70, S D=1.29), F(1,65)=2.21, p=.142$. Similarly for the nonmatching fingerprint pair, there were no main effects of context type on the latent fingerprint between those presented with context implying innocence $(M=4.54, S D=1.40)$ and implying guilt $(M=4.54, S D=1.19), F(1,65)=0.05, p=.833$, the suspect fingerprint between those presented with context implying innocence $(M=4.27, S D=1.45)$ and implying guilt $(M=3.90$, $S D=1.25), F(1,65)=2.31, p=.133$, or decisions between those presented with context implying 
innocence $(M=4.76, S D=1.19)$ and implying guilt $(M=5.00, S D=1.16), F(1,65)=1.20, p=$ .277 .

For the matching fingerprint pair again, the resulting $2 \times 2$ ANOVA also revealed no main effects of context timing on the latent fingerprint between those presented with context before ( $M$ $=3.42, S D=1.34)$ or after observation $(M=3.49, S D=1.46), F(1,65)=0.05, p=.829$, the suspect fingerprint between those presented with context before $(M=4.11, S D=1.06)$ or after observation $(M=4.04, S D=1.48), F(1,65)=0.10, p=.755$, or decisions between those presented with context before $(M=4.97, S D=1.28)$ or after observation $(M=4.98, S D=1.28)$, $F(1,65)=0.02, p=.881$. Similarly for the nonmatching fingerprint pair, there were no main effects of context timing on the latent fingerprint between those presented with context before ( $M$ $=4.60, S D=0.92)$ or after observation $(M=4.50, S D=1.53), F(1,65)=0.16, p=.692$, the suspect fingerprint between those presented with context before $(M=4.14, S D=1.17)$ or after observation $(M=4.06, S D=1.51), F(1,65)=0.51, p=.479$, or decisions between those presented with context before $(M=4.88, S D=0.96)$ or after observation $(M=4.86, S D=1.32)$, $F(1,65)=0.01, p=.916$.

Mirroring above, due to the multivariate analyses of variance not including the no context conditions, one-way analyses of variance were conducted assessing the effect of context type and timing on participants' confidence ratings regarding minutia numbers and decisions for both the matching and nonmatching fingerprint pairs. For the matching pair, the resulting ANOVA revealed no main effects of context type on the latent fingerprint, $F(2,127)=1.61, p=.203$, or the suspect fingerprint, $F(2,126)=2.25, p=.110$. However, there was a main effect of context type on decisions, $F(2,113)=3.32, p=.040$, such that participants' confidence in their decisions was higher for those presented with context implying innocence $(M=5.24, S D=1.22)$ than 
those presented with no context $(M=4.51, S D=1.32), p=.043$, mean difference $=0.72,95 \% \mathrm{CI}$ $[0.02,1.43]$. For the nonmatching pair, results revealed no main effects of context type on the latent fingerprint, $F(2,128)=0.17, p=.845$, the suspect fingerprint, $F(2,126)=1.27, p=.285$, or decisions, $F(2,120)=0.98, p=.378$.

Furthermore, for the matching pair, the resulting ANOVA revealed no main effects of context timing on the latent fingerprint, $F(2,127)=1.34, p=.267$, the suspect fingerprint, $F(2,126)=2.28, p=.107$, or decisions, $F(2,113)=1.63, p=.201$. For the nonmatching pair, results again revealed no main effects of context timing on the latent fingerprint, $F(2,128)=$ $0.22, p=.800$, the suspect fingerprint, $F(2,126)=0.53, p=.593$, or decisions, $F(2,120)=0.62, p$ $=.542$.

\section{Measures}

Data from the two personality measures (Need for Cognition scale and Compliance scale) and the demographics questionnaire were collected online through the use of Qualtrics. However, we did not include a question on Qualtrics relating to their participant number and, given two participants completed the study at the same time, it was not possible to later link the data back to the corresponding participant. Consequently, the following reported information from the two personality measures and demographics report summary ratings rather than individual differences.

Scores for the Need for Cognition scale can range from -72 to 72, with higher scores indicating higher levels of need for cognition. In the current sample, 128 participants completed the Need for Cognition scale, with scores ranging from -51 to 64 . The mean of all scores was $14.93(S D=19.14)$, demonstrating that more participants showed a tendency toward a need for cognition than those showing a tendency toward no need for cognition. As with the Need for 
Cognition scale, 128 participants completed the Compliance scale. Scores ranged from 2 to 20 out of a possible range of 1 to 20 . The mean of all scores was $10.00(S D=3.57)$; therefore, the group of participants did not show a trend toward more or less compliant behaviour.

\section{Discussion}

The current study aimed to investigate whether contextual information affects examiners' perception of fingerprint evidence and/or their decision-making process regarding whether the fingerprints are a match, nonmatch, or if it is inconclusive. It was hypothesized that the contextual information would affect both the examiner's perception of the evidence as well as their decision-making process. This hypothesis was partially supported.

\section{Overall Accuracy}

Participants demonstrated a great amount of overall accuracy. This consisted of participants making a match judgment when presented with the matching fingerprint pair or a nonmatch judgment when presented with the nonmatching fingerprint pair. It is important to note that this accuracy percentage is compared to two other options (e.g., nonmatch or inconclusive), therefore demonstrating that participants performed much better than chance in their decisions. This high amount of accuracy is surprising. Participants were trained using three short videos available to the public on YouTube, totaling to about 10 minutes in length. Regardless of this very limited training, participants were very often able to correctly identify whether the fingerprint pair was a match or nonmatch. It is possible that these rather simple videos provided participants with a significant amount of knowledge and guidance regarding how to correctly identify a matching/nonmatching pair of fingerprints. Conclusions of participants not presented with any training would also have been interesting. 
Looking specifically within fingerprint pairs, participants performed much better when examining the matching fingerprint pair than when examining the nonmatching fingerprint pair. This large difference likely speaks to the ambiguity of the fingerprints within each pair. It is likely that participants could more easily discern that the matching fingerprint pair was indeed a match while it was more difficult for them to discern that the nonmatching fingerprint pair was not a match. As described earlier, the matching fingerprint pair was created through the use of a single fingerprint (used as the suspect fingerprint within the pair) while the matching latent fingerprint was created by altering the quality of the original fingerprint to make it a darker shade, blurrier, and more difficult to discern the minutia within it. Due to participants demonstrating a greater ability to correctly identify the matching fingerprint pair, it may mean that the ambiguity created by altering the quality of this print did not equate the ambiguity existing in the nonmatching fingerprint pair.

\section{Accuracy, Decisions, and Minutia as a Function of Context}

It was hypothesized that contextual information would affect both the examiner's perception of the evidence as well as their decision-making process, and therefore, that it would affect participants' accuracy. The results concerning accuracy and context did not support this hypothesis. Participants decisions (match, nonmatch, or inconclusive) were assessed as a function of whether contextual information was given. As with accuracy, we expected participants decisions to be influenced by the contextual information provided. However, this prediction was not supported. This is parallel to our previous findings regarding participants' accuracy, demonstrating that the contextual information given had no influence on examiners' final conclusions or their accuracy. Furthermore, it was hypothesized that those presented with 
contextual information implying guilt would be more likely to conclude there is a match between fingerprints. Because context had no effect on any decisions, this hypothesis was not supported.

We know from prior studies that contextual information can affect examiners' conclusions (e.g., Dror et al., 2005, Dror et al., 2006, Kukucka \& Kassin, 2014). The current study aimed to determine what part of the examination is affected by contextual information, perception or decision-making. However, in order to determine this, the contextual information may have to demonstrate this influence on final conclusions about the evidence, to then determine where this effect took place. Though numerous studies have demonstrated the ability to influence the conclusions of laypersons trained in evidence examination by presenting simple contextual information such as the presence of a recanted confession (e.g., Kukucka, 2014; Kukucka and Kassin, 2014; Miller, 1984), the current study demonstrated no effects of contextual information on participants' conclusions. Therefore, we were unable to further investigate what part of the examination is affected by contextual information concerning the guilt or innocence of the suspect.

While it was hypothesized that contextual information would affect perception of the evidence, and therefore the numbers of minutia counted, results did not support this prediction. However, this does not necessarily mean that contextual information does not affect the perception of evidence. If results demonstrated an effect of the contextual information on participants' conclusions, we would then be able to discern if the contextual information influenced the perception of evidence specifically. However, because of the issues that related to participants not being influenced by the contextual information, no conclusions can be made regarding the impact of context on the perception of evidence. 
Even with participants being given the more explicit context information, it had no effect on decisions. It is possible that the amount or type of information provided was still not explicit or attention-getting enough to alter how participants made their decisions. For instance, if more information was provided about corroborating evidence, or the lack thereof, participants may have been more inclined to decide in favour of that information. It is also possible that participants simply did not believe the contextual information told to them; because this information came from a female around their own age, the believability that this information came from a professional source such as a prosecutor or a defence attorney may have been compromised. Furthermore, because this study took place in a lab setting on the campus of the participants' undergraduate university, participants may not have been so inclined to believe they were examining fingerprints from real criminal cases and being told information from investigators/attorneys. Therefore, it may be the case that we were not able to simulate the setting of a real forensic examination as we had hoped. Other studies have primarily conducted research by administering online, thus making this particular issue not common in the literature.

Additionally, because this information was relayed from a third party (the researcher), instead of directly from an investigator or attorney, the information's salience could have been compromised. Dror (2013) noted that experts in particular, especially those with a great amount of experience doing these examinations, are more susceptible to cognitive biases and the influence of contextual information due to the method of examining becoming more based on short-cuts and relying on past experience. Due to the participants in the current study being all newly trained individuals in fingerprint examination, it is possible that the cognitive biases they are subject to are in fact less influential than those for an expert examiner. 
Lastly, though the intention was to be consistent with the presentation of contextual information in real forensic science examination, having the context be provided by the prosecution or defence does introduce a possible confound. Because of this added factor, we also cannot conclude that findings related to the context-direction manipulation are due to the information or who it was provided by.

\section{Minutia and Accuracy}

Because it was hypothesized that contextual information would affect perception, it was predicted that the numbers of minutia counted would affect accuracy. However, this was not the case. If the accuracy of judgements relates to the perception of the fingerprint evidence, we would expect to see differences in the numbers of minutia counted. Therefore, because minutia numbers did not differ between participants that were and were not accurate in their decisions, it may imply that the decision-making phase has more of an impact on examiners' accuracy than the perception of the evidence (the individual fingerprint examination phase of the process). Thus, this may give us some insight into what part of the examination is more critical to obtaining accurate results from examiners.

\section{Accuracy and Decisions as a Function of Context Timing}

Lastly, the accuracy of judgements was assessed as a function of when contextual information was given (before observation and decision-making or after observation but before decision-making). Again, it was hypothesized that contextual information would affect both the examiner's perception of the evidence as well as their decision-making process, and therefore, participants' accuracy and decisions. Results regarding accuracy and context timing partially supported this hypothesis. Parallel to this finding, results regarding participants' decisions and context timing partially supported this hypothesis. Participants' accuracy (accurate or inaccurate) 
and their decisions (march, nonmatch, or inconclusive) when reviewing the nonmatching fingerprint pair were affected by contextual information when it was presented directly before decision-making.

These findings speak to what the contextual information is affecting in the examination. Though the actual case information given demonstrated no effect on participants' judgements, this finding demonstrates that the time at which context is presented can have its own effect on examiners' judgements, over and above the type of information given. Because accuracy was negatively affected when context was presented after observation but before decision-making, more so than when context was presented before both observation and decision-making, it is reasonable to conclude that information given closer to the decision-making phase (after examination of the individual fingerprints is complete) has a more substantial impact on conclusions than when presented prior to the start of the examination. This could mean that the information is having a distracting effect on the decision-making process and compromising examiners' ability to make accurate judgements.

\section{Match-Confidence Composite Score}

Match-confidence composite scores were created to assess any differences between the contextual information conditions. It was hypothesized that participants who were presented with contextual information implying the suspect's guilt would have higher composite scores than those in the other context conditions. This hypothesis was not supported. For both sets 1 and 2, match-confidence composite scores did not differ between participants presented with information implying guilt, innocence, or presented with no context. Because of the earlier finding regarding no effect on decisions as a function of contextual information in the current 
sample, it is to be expected that match-confidence composite scores would also not differ as a function of context.

\section{Implications}

Ultimately, because the contextual information given did not have an effect on decisions, accuracy, or minutia, we were unable to discern whether contextual information affects the perception of evidence, the decision-making process, or both. However, what can be taken from this research is that regarding the timing of the presentation of contextual information. This study demonstrated that the time at which context is presented in the examination can affect participants' accuracy and decisions. Furthermore, when assessing differences in minutia numbers between participants who were accurate and inaccurate in their decisions, no differences were found. Both of these results may tell us about the aspect of the examination that is more critical in gaining accurate conclusions and, if context is being presented, when it should be presented in order for it to have less of an impact on examiners' conclusions.

Because accuracy was negatively affected when context was presented just before decision-making, rather than when presented prior to the examination, it is reasonable to conclude that information given closer to the decision-making phase (after examination of the individual fingerprints is complete) has a more substantial impact on conclusions. This could mean that the information is having a distracting effect on the decision-making process, regardless of the content of information, and compromising examiners' ability to make accurate

judgements. Furthermore, because no differences were found in the numbers of minutia counted (our perception variable) between participants who were and were not accurate in their decisions, this also tells us that the perception element, the individual fingerprint inspection phase of the examination, may not be as critical as the decision-making phase. What this tells us is that, if 
contextual information is going to be presented to examiners, it is best for it to be presented prior to examinations, as this may have less of an influence on conclusions than if presented during the examination and closer to the decision-making phase.

These findings suggest that the decision-making phase has more of an impact on examiners' conclusions than the perception of the evidence. This would also mean that subjectivity in the examination is even higher than previously thought. If the subjective decision-making regarding whether two fingerprints are sufficiently similar, a term yet to be defined (Kassin et al., 2013a), is having a greater influence on final decisions than the visual perception of the evidence, then this demonstrates how open to interpretation evidence examination is. Furthermore, this demonstrates the need for strict definitions and protocols surrounding this decisions-making process and for the explicit definition of what a match finding should consist of. However, it is important to keep in mind that the findings regarding the influence of the time context was given on decisions and accuracy only concerned the examination of the nonmatching fingerprint pair. Therefore, more research is needed to investigate whether this effect would stand with matching fingerprint pairs as well and if it holds for nonmatching fingerprint pairs.

\section{Limitations and Future Directions}

First, because contextual information had no demonstrable effect on decisions or the accuracy of these decisions, it may be that our context manipulation was not strong enough. it is possible that the context given should have included more information regarding other evidence against or supporting the innocence of the defendant. Additionally, because this information was provided by a female of a similar age as participants, instead of a professional investigator or attorney, and due to the environment being in a lab at the participant's university, it is possible 
that individuals were less likely to believe the information provided. Future studies should ensure that contextual information is as salient as possible by providing information in a professional manner and relay this information in a way that simulates a real forensic examination.

Dror and Hampikian (2011) found a significant impact on conclusions when there was a need for corroborating evidence. That is, when participants felt there was a great need for the evidence at hand to support other evidence for or against the suspect, examiners' conclusions were more likely to be in favour of these other findings. Therefore, future studies should not only include information about other evidence for or against the suspect, but also create the sense that there is need for a particular finding from the examiner. Saks and colleagues (2003) noted that transmittal letters are often included with evidence sent for examination by investigators, including extraneous case information. This form of relaying information from investigators could be included in future research, as opposed to verbally conveying context, to create a more realistic simulation. This would also eliminate the issue of the researcher negatively impacting the believability of the contextual information, as it may be more believable that this information came from a valid source if in the form of a professional document.

Other than the limitations regarding the content and presentation of contextual information, there are those relating to the significant findings. Though this study found accuracy and decisions to be impacted by when context was presented, this was for the nonmatching fingerprint pair only. Future studies should further investigate whether this is the case for matching fingerprint pairs as well. Additionally, based on these findings, future research should investigate the impact of information on the decision-making phase of examination in particular. Lastly, as the current study intended, future research should aim to 
investigate what part of the examination is being affected when examiners are told information implying the suspect's guilt or innocence.

\section{Conclusion}

The National Academy of Sciences (2009) highlighted the scarcity of research pertaining to cognitive biases within forensic science and stated the need for an expansion of this research. The current study aimed to fill the gap in literature concerning the specific part of examinations that is affected by the presentation of contextual information. Though the specific context provided did not have an overall effect on examiners' conclusions, we did find that the timing of this context could interfere with the decision-making process, in that context presented just before decisions-making can lead to more inaccurate judgements. Our findings suggest that context has less of an impact on examiners' judgments when presented prior to the presentation of fingerprints. If contextual information is going to be presented to examiners, this type of information is crucial in order to identify how we can alter the presentation of this information so that it has a minimal impact on examiners' judgments and thus leads to the most accurate conclusions possible. 


\section{Appendix A}

Demographics Questionnaire:

What is your age?

What is your gender?

Male

Female

Trans

Other, please specify

Please specify your race.

White

Chinese

South Asian (e.g., East Indian, Pakistani, Sri Lankan, etc.)

Black

Filipino

Latin American

Southeast Asian (e.g., Vietnamese, Cambodian, Malaysian, Laotian, etc.)

Arab

West Asian (e.g., Iranian, Afghan, etc.)

Korean

Japanese

Aboriginal Canadian/First Nations

Metis

Other, please specify 


\section{Appendix B}

Please indicate the extent to which you agree or disagree with each of the following statements using the scale below.

$\begin{array}{lllllllll}-4 & -3 & -2 & -1 & 0 & 1 & 2 & 3 & 4\end{array}$

Very Strongly Moderately Slightly Neither Slightly Moderately Strongly Very strongly disagree disagree disagree agree nor agree agree agree strongly disagree disagree agree

1) I would prefer complex problems to simple problems.

2) I like to have the responsibility of handling a situation that requires a lot of thinking.

*3) Thinking is not my idea of fun.

*4) I would rather do something that requires little thought than something that is sure to challenge my thinking abilities.

*5) I try to anticipate and avoid situations where there is likely a chance I will have to think in depth about something.

6) I find satisfaction in deliberating hard and for long hours.

*7) I only think as hard as I have to.

*8) I prefer to think about small, daily projects rather than long-term ones.

*9) I like tasks that require little thought once I"ve learned them.

10) The idea of relying on thought to make my way to the top appeals to me.

11) I really enjoy a task that involves coming up with new solutions to problems.

*12) Learning new ways to think doesn"t excite me very much.

13) I prefer my life to be filled with puzzles that I must solve.

14) The notion of thinking abstractly is appealing to me. 
15) I would prefer a task that is intellectual, difficult, and important to one that is somewhat important but does not require much thought.

*16) I feel relief rather than satisfaction after completing a task that required a lot of mental effort.

*17) It is enough for me that something gets the job done; I don "et care how or why it works.* 18) I usually end up deliberating about issues even when they do not affect me personally. $*=$ Item is reverse-scored. 


\section{Appendix C}

I. I give in easily to people when I am pressured

2. I find it very difficult to tell people when I disagree with them

3. People in authority make me feel uncomfortable and uneasy

4. I tend to give in to people who insist that they are right

5. I tend to become easily alarmed and frightened when I am in the company of people in authority

6. I try very hard not to offend people in authority

7. I would describe myself as a very obedient person

8. I tend to go along with what people tell me even when I know that they are wrong

9. I believe in avoiding rather than facing demanding and frightening situations

10. I try to please others

11. Disagreeing with people often takes more time than it is worth

12. I generally believe in doing as I am told

13. When I am uncertain about things I tend to accept what people tell me

14. I generally try to avoid confrontation with people

15. As a child I always did what my parents told me

16. I try hard to do what is expected of me

17. I am not too concerned about what people think of me

18. I strongly resist being pressured to do things I don't want to do

19. I would never go along with what people tell me in order to please them

20. When I was a child I sometimes took the blame for things I had not done 


\section{Appendix D}

Ryerson University

Consent Agreement

ANALYZING FINGERPRINT EVIDENCE: CAN YOU DO IT LIKE THE PROS?

You are being asked to participate in a research study. Before you give your consent, it is important that you read the following information and ask as many questions as necessary to be sure you understand what you will be asked to do.

\begin{tabular}{|c|c|c|c|}
\hline Investigator: & $\begin{array}{l}\text { Melanie Taylor } \\
\text { MA student } \\
\text { Department of Psychology }\end{array}$ & Supervisor: & $\begin{array}{l}\text { Dr. Tara Burke } \\
\text { Associate Professor } \\
\text { Department of Psychology }\end{array}$ \\
\hline
\end{tabular}

Purpose of the Study: The purpose of this 60 minute, 1 credit social psychological study is to compare the observations and conclusions of forensic examiners to undergraduates in analyzing fingerprint evidence in order to determine if forensic examiners are significantly better at doing these analyses. Approximately 150 Ryerson Students enrolled in PSY102 and PSY202 will participate in this study. You may be completing the study with another participant in the room. However, you will not be working together or sharing information.

Description of the Study: If you decide to participate in this research, you will be asked to do the following: You will first watch three short videos describing how to analyze fingerprint evidence. You will then be presented with four different fingerprint pairs to analyze and make judgements about. After this, you will answer some brief questionnaires assessing various aspects of your personality. You will be given $1 \%$ course credit for participating.

What is Experimental in this Study: From a technical or procedural point of view, part of this study is considered "experimental," because by following the procedure described above, the study examines the impact of one variable (called the "independent variable") on another variable ("called the dependent variable"). You will be given more information about the independent and dependent variables in this study at the end of today's session.

Risks or Discomforts: This is a minimal risk study. Any discomfort is expected to be temporary and not greater than you might experience in a typical day. Occasionally people may feel uncomfortable when answering questionnaires that ask about attitudes toward personal issues. While we do not anticipate that any of the questions you will be responding to are of this nature, if any aspect of this study makes you feel uncomfortable, you may choose not to answer certain questions, or to withdraw from the study at any time and still receive your credit.

Benefits of the Study: We anticipate that you will benefit from this study by learning more about how evidence is presented in the courtroom and the steps involved in social psychological research. When the session is over, we will describe the purpose and hypotheses of the study to you in more detail. Also, once we have completed data collection and analyses (end of Fall 2017) you are welcome to contact the researchers via email to view the results.

Confidentiality: Your responses in this research will be confidential and anonymous; your name will not be linked to your responses. Any materials that include your name - this consent form-will be stored separately from any other data for a minimum of 5 years. Physical materials will be stored in a locked 
filing cabinet in a locked room, and computer files will be stored on password-protected computers. Only the investigator and other Psychology and Law lab personnel will have access to this information. Your responses on any questionnaires will be identified only by a participant number assigned to you by the researchers. This number cannot later be used to identify you and is unrelated to your Sona ID or student number. The online questionnaire is hosted by Qualtrics, which is an American company. Consequently, Qualtrics or USA authorities may access survey data in some forms (e.g., aggregate usage information) and under strict policies. Qualtrics employs a variety of security features to make sure that the data collected are not accessible by outside bodies. More information on Qualtrics' security systems can be viewed here: https://www.qualtrics.com/security-statement/. Information regarding their protective privacy policy is available here: https://www.qualtrics.com/privacy-statement/ . Although Qualtrics usually stores IP address data, we have deactivated that function for this study

Incentives to Participate: You will receive 1\% course credit for your Introductory Psychology course. If you would prefer to walk through of the study - that is, if you would like to observe the research process but not provide any personal data - you will still be given $1 \%$ course credit. Note that while you can take part in as many psychology research studies as you wish, you cannot exceed the maximum allotted course credits, as set by your Introductory Psychology course.

Voluntary Nature of Participation: Participation in this study is voluntary. Your choice of whether or not to participate will not influence your future relations with Ryerson University. If you decide to participate, you are free to withdraw your consent and to stop your participation at any time. Should you withdraw from the study, or if you choose not to answer some questions, you will still be given your $1 \%$ course credit (provided you have not already received the maximum allotted for research participation for the term).

Dissemination of Results: Anonymized data may be provided to other researchers for the purpose of study or verification of results; any data that is shared will NOT include the names of ANY participants. It is possible that a third party (e.g., graduate students, senior undergraduate students) may have access to the data for a purpose that was not originally identified in this study. As well, results may be shared with others at scholarly meetings or as part of published papers. However, all information will be presented in aggregate form. That is, none of your individual information will be identifiable in any way.

Questions about the Study: If you have any questions about the research now, please ask. If you have questions later about the research, you may contact.

Melanie Taylor, 416-979-5000 x2190, melanie.taylor@psych.ryerson.ca

Dr. Tara Burke, 416-979-5000, ex. 6519, tburke@ psych.ryerson.ca

If you have questions regarding your rights as a human subject and participation in this study, please contact the Ryerson University Research Ethics Board: Ryerson Ethics Board, c/o Office of the Vice President, Research and Innovation, Ryerson University 350 Victoria Street Toronto, ON M5B 2K3, 416979-5042, rebchair@ ryerson.ca

If you have questions about your participation in the Introductory Psychology Participant Pool, please contact thepool@psych.ryerson.ca 
Agreement: Your signature below indicates that you have read the information in this agreement and have had a chance to ask any questions you have about the study. Your signature also indicates that you agree to be in the study and have been told that you can change your mind and withdraw your consent to participate at any time.

Name of Participant (please print)

Signature of Participant

Date

Signature of Investigator

Date 


\section{Appendix E}

How many minutia did you count in the latent (first) fingerprint?

How confident are you that you counted the correct number of minutia in this print?
1
2
3
4
5
6

Not at all

Neither

confident

$$
\begin{gathered}
\text { confident nor } \\
\text { unconfident }
\end{gathered}
$$

How many minutia did you count in the suspect (second) fingerprint?

How confident are you that you counted the correct number of minutia in this print?

1

Not at all

confident
2

3

4

Neither confident nor unconfident
6

7

Extremely confident

Please circle one to specify your final judgement of the two fingerprints.

$\begin{array}{ccc}\begin{array}{c}\text { Identification } \\ (\text { match })\end{array} & \begin{array}{c}\text { Exclusion } \\ \text { (nonmatch) }\end{array} & \begin{array}{c}\text { Inconclusive } \\ \text { (neither match nor nonmatch) }\end{array}\end{array}$

If your final judgement was either an identification (match) or exclusion (nonmatch), rate your confidence in your decision (if your final judgement was inconclusive please exclude this question).

1

2

3

4

5

Neither

confident nor

unconfident
Extremely

confident 


\section{Appendix F}

\section{Debriefing - Analyzing fingerprint evidence: Can you do it like the pros?}

Wrongful convictions occur when innocent people are convicted of crimes they did not commit. Errors in forensic science is one factor that has been shown to contribute to wrongful convictions and has contributed to the conviction of nearly half of wrongful conviction cases (Innocence Project, 2017). There are many studies that demonstrate how one's knowledge, beliefs, and motivations influence how he/she interprets information or visual stimuli; this includes how forensic examiners interpret forensic science evidence (such as fingerprints; Charman, Gregory, \& Carlucci, 2009). Typically in fingerprint and other forensic science examinations, examiners are told a great deal of information by the hiring body regarding the case and the suspect (Saks, Risinger, Rosenthal, \& Thompson, 2003). This information can include other types of evidence investigators have found against the suspect as well as an investigator's belief in the suspect's guilt, often leading the forensic examiner to also believe the suspect is guilty (Saks et al., 2003). When the forensic examiner believes the suspect is guilty or simply is aware of other evidence against the suspect, this has been shown to influence the examiner's conclusions about the forensic evidence (for instance, whether the suspect's fingerprint matches the fingerprint found at the crimescenel; Charman et al., 2009).

Though this influence on forensic examinations has been demonstrated through previous research, we have yet to understand more about how this bias operates and how such case information can influence the examiner's conclusions. What this study intends to investigate is whether the case information is influencing an examiner's perception of the visual stimuli (i.e., what is perceived from looking at the fingerprints), the later decision-making process about whether the two fingerprints can be considered a match, or both processes. All participants were asked to watch the same videos about how to examine fingerprints. However, participants were presented with case information before observation of the fingerprints, after observation of the fingerprints, or after making a decision about the fingerprints (this group was asked to go back and confirm their answers after the information was presented; an independent variable). Also, participants were either presented with case information suggesting the suspect's innocence or the suspect's guilt (a second independent variable). We asked you to provide the numbers of minutia, or points of reference, observed in each fingerprint, your final conclusion (match, nonmatch, or inconclusive), and your confidence ratings regarding these decisions (the dependent variables).

Our first hypothesis is that the case information will affect both the examiner's perception of the fingerprints as well as their decision-making process.

Our second hypothesis is that participants who were presented with case information suggesting the suspect's guilt (rather than innocence) will be more likely to conclude a match between fingerprints.

Our third hypothesis is that participants who were presented with case information after making a decision about the fingerprints will be more likely to conclude a match when they are asked to later confirm their answers than during their first examination. 
The information you provided during this study may allow us to further demonstrate the need for change in forensic examination procedure, possibly leading to changes in actual examination procedure within our justice system.

If you know of others who will be participating in this experiment, please refrain from discussing the study with them. We do not want our future participants to be aware of the exact procedures and expected findings.

Thank you for participating in our research. If you have any questions about the study, please contact:

Melanie Taylor

Tara Burke, Ph.D.

melanie.taylor@psych.ryerson.ca

tburke@psych.ryerson.ca

416-979-5000 x2190

416-979-5000 x6519

\section{If you have any questions about receiving your Psychology 102/202 credit participation please contact: thepool@ psych.ryerson.ca}

If you have questions regarding your rights as a human subject and participation in this study, please contact the Ryerson University Research Ethics Board: Ryerson Ethics Board, c/o Office of the Vice President, Research and Innovation, Ryerson University 350 Victoria Street Toronto, ON M5B 2K3, 416979-5042, rebchair@ ryerson.ca

\section{References:}

Charman, S. D., Gregory, A. H., \& Carlucci, M. (2009). Exploring the diagnostic utility of facial composites: Beliefs of guilt can bias perceived similarity between composite and suspect. Journal of Experimental Psychology: Applied, 15, 76-90. doi:

$10.1037 / \mathrm{a} 0014682$

Innocence Project. (2017). Misapplication of Forensic Science. Retrieved from https://www.innocenceproject.org/causes/misapplication-forensic-science/

Saks, M. J., Risinger, D. M., Rosenthal, R., \& Thompson, W. C. (2003). Context effects in forensic science: A review and application of the science of science to crime laboratory practice in the United States. Science \& Justice, 43, 77-90. doi: 10.1016/S13550306(03)71747-X 


\section{References}

Alpert, G. P. (1979). Inadequate defense counsel: An empirical analysis of prisoners' perceptions. American Journal of Criminal Law, 7, 1-22.

Balcetis, E. \& Dunning, D. (2006). See what you want to see: Motivational influences on visual perception. Journal of Personality and Social Psychology, 91, 612-625. doi: 10.1037/0022-3514.91.4.612

Balcetis, E. \& Dunning, D. (2010). Wishful seeing: More desired objects are seen as closer. Psychological Science, 21, 147-152. doi: 10.1177/0956797609356283

Braly, K. W. (1933). The influence of past experience in visual perception. Journal of Experimental Psychology, 16, 613-643.

Bressan, P. \& Dal Martello, M. F. (2002). Perceived resemblance and the belief in genetic relatedness. Psychological Science, 13, 213-218.

Bruner, J. S. \& Goodman, C. C. (1947). Value and need as organizing factors in perception. The Journal of Abnormal and Social Psychology, 42, 33-44. doi: 10.1037/h0058484

Bruner, J. S., \& Postman, L. (1949). On the perception of incongruity: A paradigm. Journal of personality, 18, 206-223.

Centurion: Our Mission. (2016). Retrieved from http://centurion.org/about-us/

Charlton, D., Fraser-Mackenzie, P. A. F., \& Dror, I. E. (2010). Emotional experiences and motivating factors associated with fingerprint analysis. Journal of Forensic Sciences, 55, 385-393. doi: 10.1111/j. 1556-4029.2009.01295.x

Charman, S. D., Gregory, A. H., \& Carlucci, M. (2009). Exploring the diagnostic utility of facial composites: Beliefs of guilt can bias perceived similarity between composite and suspect. Journal of Experimental Psychology: Applied, 15, 76-90. doi: 10.1037/a0014682 
Darley, J. M. \& Gross, P. H. (1983). A hypothesis-confirming bias in labeling effects. Journal of Personality and Social Psychology, 44, 20-33.

Dror, I. (2013). The ambition to be scientific: human expert performance and objectivity. Science and Justice, 53, 81-82.

Dror, I. E., Champod, C., Langenburg, G., Charlton, D., Hunt, H., \& Rosenthal, R. (2011).

Cognitive issues in fingerprint analysis: Inter- and intra-expert consistency and the effect of a 'target' comparison. Forensic Science International, 208, 10-17. doi:

10.1016/j.forsciint.2010.10.013

Dror, I. E., Charlton, D., \& Péron, A. E. (2006). Contextual information renders experts vulnerable to making erroneous identification. Forensic Science International, 156, 7578. doi: 10.1016/j.forsciint.2005.10.017

Dror, I. E., \& Cole, S. A. (2010). The vision in "blind" justice: Expert perception, judgment, and visual cognition in forensic pattern recognition. Psychonomic Bulletin \& Review, 17, 161167. doi: 10.3758/PBR.17.2.161

Dror, I. E. \& Hampikian, G. (2011). Subjectivity and bias in forensic DNA mixture interpretation. Science \& Justice, 51, 204-208. doi: 10.1016/j.scijus.2011.08.004

Dror, I. E., Peron, A. E., Hind, S. L., \& Charlton, D. (2005). When emotions get the better of us: The effect of contextual top-down processing on matching fingerprints. Applied Cognitive Psychology, 19, 799-809. doi: 10.1002/acp.1130

Dror, I. E., Wertheim, K., Fraser-Mackenzie, P., \& Walajtys, J. (2012). The impact of humantechnology cooperation and distributed cognition in forensic science: biasing effects of AFIS contextual information on human experts. Journal of Forensic Sciences, 57, 343352. doi: 10.1111/j.1556-4029.2011.02013.x 
Edwards, H., \& Gotsonis, C. (2009). Strengthening forensic science in the United States: A path forward. Statement before the United State Senate Committee on the Judiciary.

Gottschaldt, K. (1926). On the influence of experience on the perception of figures. Psychological Research, 8, 261-317.

Garrett, B. [The Innocence Project]. (2011, July 15). Getting it right: Informants [Video file]. Retrieved from http://www.innocenceproject.org/causes/incentivized-informants/

Garrett, B. L. \& Neufeld, P. J. (2009). Invalid forensic science testimony and wrongful convictions. Virginia Law Review, 95, 1-97.

Hagan, W. E. (1894). A treatise on disputed handwriting and the determination of genuine from forged signatures. New York, NY: Banks \& Brothers.

Hampikian, G., West, E., \& Akselrod, O. (2011). The genetics of innocence: Analysis of 194 U.S. DNA exonerations. Annual Review of Genomics and Human Genetics, 12, 97-120. doi: 10.1146/annurev-genom-082509-141715

Hodge, E. (1988). Guarding against error. Journal of the Association of Firearms \& Toolmark Examiners, 20, 290-292.

Innocence Project. (2018). False Confessions or Admissions. Retrieved from https://www.innocenceproject.org/causes/false-confessions-admissions/

Innocence Project. (2018). Inadequate Defense. Retrieved from https://www.innocenceproject.org/causes/inadequate-defense/

Innocence Project. (2018). Misapplication of Forensic Science. Retrieved from https://www.innocenceproject.org/causes/misapplication-forensic-science/ Kassin, S. M., Bogart, D., \& Kerner, J. (2012). Confessions that corrupt: Evidence from the 
DNA exoneration case files. Psychological Science, 23, 41-45. doi:

$10.1177 / 0956797611422918$

Kassin, S. M., Drizin, S. A., Grisso, T., Gudjonsson, G. H., Leo, R. A., \& Redlich, A. D. (2010). Police-induced confessions: Risk factors and recommendations. Law and Human Behavior, 34, 3-38. doi: 10.1007/s10979-009-9188-6

Kassin, S. M., Dror, I. E., \& Kukucka, J. (2013a). The forensic confirmation bias: Problems, perspectives, and proposed solutions. Journal of Applied Research in Memory and Cognition, 2, 42-52. doi: 10.1016/j.jarmac.2013.01.001

Kassin, S., Fein, S., Markus, H. R., \& Burke, T. M. (2013b). Social psychology (2nd ed.). Toronto, ON: Nelson Education.

Kukucka, J. (2014). An investigation of factors that create and mitigate confirmation bias in judgments of handwriting evidence. Retrieved from UMI Dissertation Publishing. (UMI 3623628)

Kukucka, J. \& Kassin, S. M. (2014). Do confessions taint perceptions of handwriting evidence? An empirical test of the forensic confirmation bias. Law and Human Behavior, 38, 256270.

Kukucka, J., Kassin, S. M., Zapf, P. A. \& Dror. I. E. (2017). Cognitive bias and blindness: A global survey of forensic science examiners. Journal of Applied Research in Memory and Cognition, 6, 452-459. doi: 10.1016/j.jarmac.2017.09.001

Natapoff, A. (2006). Beyond unreliable: How snitches contribute to wrongful convictions. Golden Gate University Law Review, 37, 1-24.

National Academy of Sciences. (2009). Strengthening Forensic Science in the United States: A Path Forward. Washington, DC: National Academies Press. 
Lange, N. D., Thomas, R. P., Dana, J., \& Dawes, R. M. (2011). Contextual biases in the interpretation of auditory evidence. Law and Human Behavior, 35, 178-187. doi: $10.1007 / \mathrm{s} 10979-010-9226-4$

Leo, R. A. (2009). False confessions: Causes, consequences, and implications. Journal of the American Academy of Psychiatry and the Law, 37, 332-43.

Miller, L. S. (1984). Bias among forensic document examiners: A need for procedural changes. Journal of Police Science and Administration, 12, 407-411.

Miller, L. S. (1987). Procedural bias in forensic science examination of human hair. Law and Human Behavior, 11, 157-163.

Peterson, J. L., Mihajlovic, S., \& Gilliland, M. (1984). Forensic evidence and the police: The effects of scientific evidence on criminal investigations. Washington, DC: National Institute of Justice.

Saks, M. J., Risinger, D. M., Rosenthal, R., \& Thompson, W. C. (2003). Context effects in forensic science: A review and application of the science of science to crime laboratory practice in the United States. Science \& Justice, 43, 77-90. doi: 10.1016/S13550306(03)71747-X

Steblay, N., Dysart, J., Fulero, S., \& Lindsay, R. C. L. (2003). Eyewitness accuracy rates in police showup and lineup presentations: A meta-analytic comparison. Law and Human Behavior, 27, 523-540. doi: 10.1023/A:1025438223608

Stoel, R. D., Dror, I. E., \& Miller, L. S. (2014). Bias among forensic document examiners: Still a need for procedural changes. Australian Journal of Forensic Sciences, 46, 91-97. doi: $10.1080 / 00450618.2013 .797026$

Tversky, A., \& Kahneman, D. (1974). Judgment under uncertainty: Heuristics and biases. 
Science, 185, 1124-1131. doi: 10.1126/science.185.4157.1124

Zapf, P. A. \& Dror, I. E. (2017). Understanding and mitigating bias in forensic evaluation: Lessons from forensic science. International Journal of Forensic Mental Health, 16, 227238. doi: 10.1080/14999013.2017.1317302

Zapf, P. A., Kukucka, J., Kassin, S. M., \& Dror, I. E. (2018). Cognitive bias in forensic mental health assessment: Evaluator beliefs about its nature and scope. Psychology, Public Policy, and Law, 24, 1-10. doi: 10.1037/law0000153 